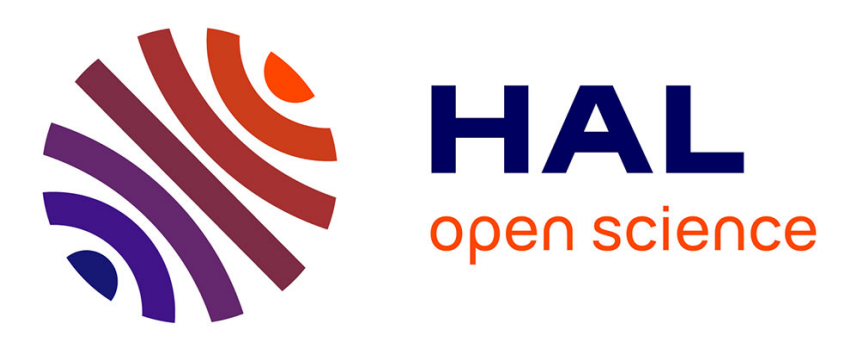

\title{
Conditional expected utility criteria for decision making under ignorance or objective ambiguity
}

Nicolas Gravel, Thierry Marchant, Arunava Sen

\section{To cite this version:}

Nicolas Gravel, Thierry Marchant, Arunava Sen. Conditional expected utility criteria for decision making under ignorance or objective ambiguity. Journal of Mathematical Economics, 2018, 78, pp.7995. 10.1016/j.jmateco.2018.08.001 . hal-01988972

\section{HAL Id: hal-01988972 \\ https://hal-amu.archives-ouvertes.fr/hal-01988972}

Submitted on 13 Mar 2020

HAL is a multi-disciplinary open access archive for the deposit and dissemination of scientific research documents, whether they are published or not. The documents may come from teaching and research institutions in France or abroad, or from public or private research centers.
L'archive ouverte pluridisciplinaire HAL, est destinée au dépôt et à la diffusion de documents scientifiques de niveau recherche, publiés ou non, émanant des établissements d'enseignement et de recherche français ou étrangers, des laboratoires publics ou privés. 


\section{amse}

école d'économie d'aix-marseille

aix-marseille school of economics

\section{Working Papers / Documents de travail}

Conditional Expected Utility Criteria for Decision Making under Ignorance or Objective Ambiguity

Nicolas Gravel

Thierry Marchant

Arunava Sen 


\title{
Conditional Expected Utility Criteria for Decision Making under Ignorance or Objective Ambiguity
}

\author{
Nicolas Gravel, Thierry Marchant ${ }^{\dagger}$ and Arunava Sen $^{\ddagger}$ \\ April 6th, 2016 \\ Keywords: Ignorance, Ambiguity, Conditional Probabilities, \\ Expected Utility, Ranking Sets, axioms \\ JEL classification numbers: D80, D81.
}

\begin{abstract}
We provide an axiomatic characterization of a family of criteria for ranking completely uncertain and/or ambiguous decisions. A completely uncertain decision is described by the set of all its consequences (assumed to be finite). An ambiguous decision is described as a finite set of possible probability distributions over a finite set of prices. Every criterion in the family compares sets on the basis of their conditional expected utility, for some probability function taking strictly positive values and some utility function both having the universe of alternatives as their domain.
\end{abstract}

\section{Introduction}

Suppose that a (public) decision maker examines the possibility of adopting an economically costly regulation that would limit carbon emissions in the next 50 years with the aim of preventing global warming. The decision maker is uncertain about the impact of carbon emission on the average earth temperature and tries to get evidence from the best scientists and available models about this. For instance, the decision maker could obtain in Meinshausen, Meinshausen, Hare,

\footnotetext{
*Aix-Marseille University (Aix-Marseille School of Economics), CNRS \& EHESS, Centre de la Vieille Charité, 2, rue de la Charité, 13002 Marseille, France, nicolas.gravel@univ-amu.fr

${ }^{\dagger}$ Ghent University, Department of Data analysis, H. Dunantlaan 1, B-9000 Gent, Belgium, Thierry.Marchant@UGent.be

$\ddagger$ Indian Statistical Institute, 7, Shaheed Jit Singh Marg, New Delhi, 110016 India, asen@isid.ac.in.
} 
Raper, Frieler, Knutti, Frame, and Allen (2009) the collection of estimated distributions of increase in earth temperature (above the pre-industrial level) that would result from doubling the amount of carbon in the atmosphere depicted in Figure 1. The decision maker could possibly obtain similar distributions of increases in the earth temperature for alternative scenarios of variations of carbon emissions, and base the regulation policy on the information provided by those alternative collections of distributions. This is a an example of a decision taken

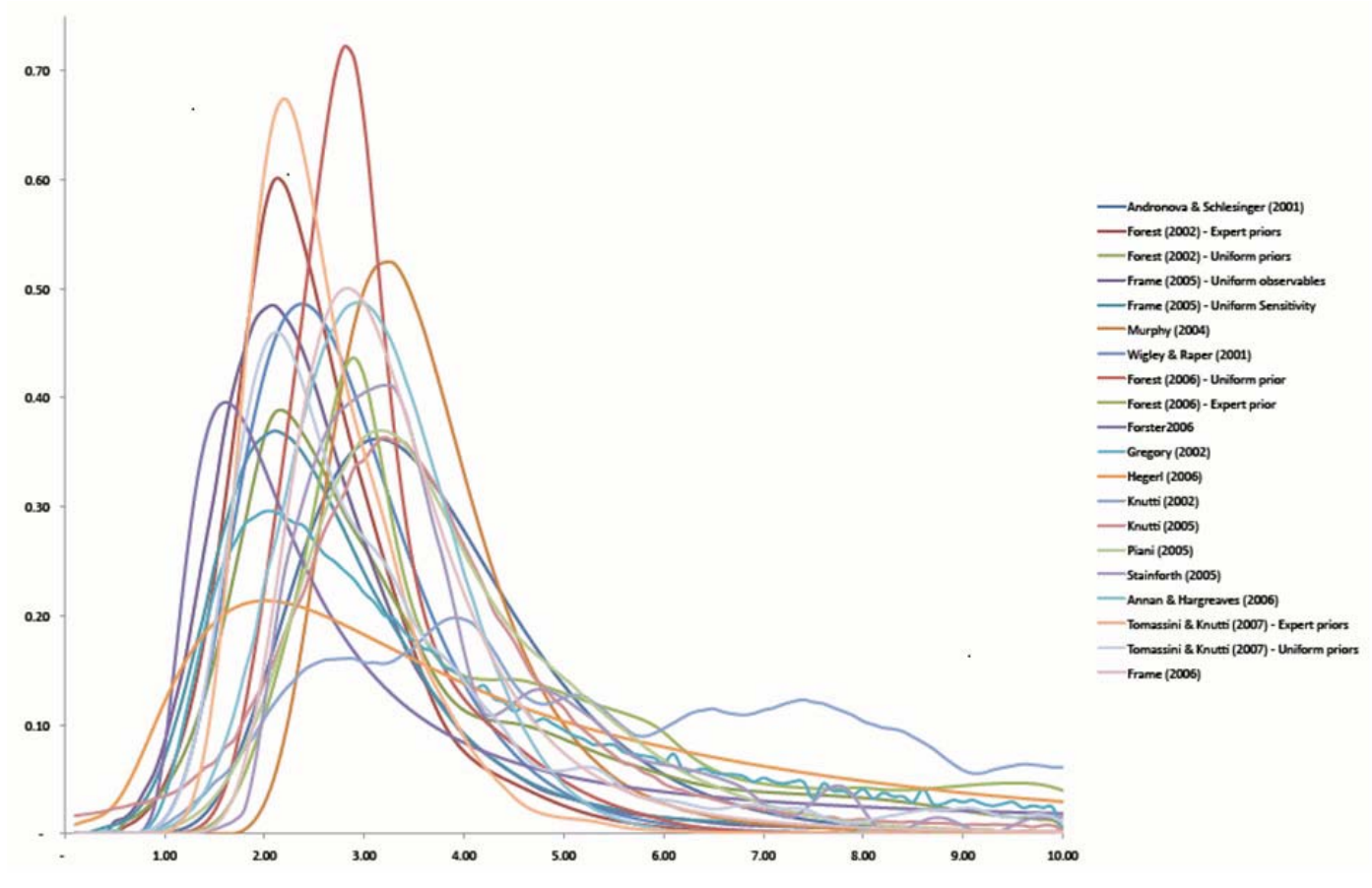

Figure 1: Estimated distributions of the increase in the Earth temperature in the next 50 years (source: Meinshausen, Meinshausen, Hare, Raper, Frieler, Knutti, Frame, and Allen (2009))

under objective ambiguity. There is ambiguity because the (probabilistic) knowledge required to take the decision is not unique. As shown on Figure 1, there are several estimates of the distributions of increase of the earth temperature. Some of them are imprecise and exhibit a large discrepancy in the predicted rises of temperature. Others are more concentrated around their "central tendency". The decision maker has no additional a priori knowledge that would enable a further discrimination between these different estimates. The ambiguity is, however, objective in the sense that these probability distributions are given to the decision maker by credible - here scientific - sources that he/she has all the reasons to believe. Other examples of decisions involving objective ambiguity include those involved in the well-known Ellsberg (1961) paradox or 
the excellent one, provided by Ahn (2008), of an ill patient having to choose between two medical treatments associated with ambiguous evidence on their probability of survival.

From a formal point of view, deciding under objective ambiguity involves comparing sets of possible probability distributions such as that described in Figure 1. It differs to that extent from decision making under (subjective) ambiguity studied in an important literature (see e.g. Epstein and Zhang (2001), Ghirartado and Marinaccin (2002), Ghirartado, Maccheroni, and Marinacci (2004), Klibanoff, Marinacci, and Mukerji (2005), Segal (1987) or Segal (1990)) that describes decisions as Savagian acts. Recall that the later are functions from a set of (mutually exclusive) states of nature - that can be enriched to lotteries $\grave{a}$ la Anscombe and Aumann (1963) - into a set of consequences. Describing decisions as Savagian acts imposes a mathematical structure that may not always be present in actual decision making processes. For instance, the public decision maker who is given the probability distributions of figure 1 is unlikely to have clear ideas - if any at all - on the "states of natures" that have generated these probabilities, or those that produce the various average temperature levels observed on the Earth surface in the next 50 years. On the other hand, such a decision maker can very well understand that a given global warming policy be associated with a collection of different probability distributions concerning a consequence of interest - for instance the average earth temperature. Additional justifications for describing decision making under objective ambiguity in terms of set rankings can be found in Ahn (2008) or Olszewski (2007).

Ranking sets of objects describe also the decision making process in situations of "ignorance" or "radical uncertainty - as these are sometimes called. In these situations, an element of a set is interpreted as a "certain" consequence that the decision associated to that set can have. The literature on ignorance has given rise also to a significant literature surveyed, for instance, in Barberà, Bossert, and Pattanaik (2004). Most of the criteria for decision making studied in this literature are based on the best and the worst consequences of the decisions or on associated lexicographic extensions. ${ }^{1}$ There are two obvious limitations of such "extremist" rankings. The first is that it is natural to believe (in line with various "expected utility" hypotheses) that decision makers are concerned with "averages" rather than "extremes". For instance, suppose that $A$ is a decision under ignorance that can result in earning either $\$ 1$ or $\$ 1000$ 000 while $B$ is an alternative decision that can lead to any integer amount of money lying between $\$ 0$ and $\$ 999$ 999. Since the extreme earnings associated to $A$ are strictly greater than those of $B$, "extremist" criteria based on the min or the max will favor the former over the latter. However, a convincing case can be made for $B$ over $A$ on the grounds that, on "average" a larger gain is likelier in the former. A second drawback of "extremist" rankings is that they do not allow for a diversity of attitudes toward ignorance across decision makers. In situations where decisions have only monetary consequences and all decision makers prefer more money to less, they will all have identical rankings over

\footnotetext{
${ }^{1}$ Notable exceptions are Baigent and Xu (2004) and Nitzan and Pattanaik (1984).
} 
decisions under positional rules such as maximin, maximax, leximin and so on. This is unsatisfactory since the fact that decision makers have the same preference over certain outcomes should not imply that they have the same attitude toward ignorance. ${ }^{2}$

In this paper, we pursue the line of inquiry of Gravel, Marchant, and Sen (2012) by providing an axiomatic characterization of a family of rankings of sets of objects that applies to decision making under either objective ambiguity - if the elements in the sets are probability distributions - or ignorance if the elements are ultimate consequences. Contrary to the "extremist" criteria considered in the literature on ignorance, the criteria that we examine can all be though of as "smooth" averages of values attached by the decision maker to the probability distributions (in the objective ambiguity setting) or the certain consequences (in the ignorance setting) associated to a particular decision. In Gravel, Marchant, and Sen (2012), we characterize the family of rankings of all finite subsets of a rich universe that can be thought of as resulting from the following two-step procedure. In the first step, all conceivable probability distributions (objective ambiguity) or certain consequences (ignorance) are evaluated by some (utility) function. In the second step, decisions are compared on the basis of their expected utility (given the function chosen in the first step) under the (uniform) assumption that all probability distributions (objective ambiguity) or consequences (ignorance) of a decision are equally likely. We call "Uniform Expected Utility" (UEU) any such ranking of sets. For example, if our public decision maker was using a UEU criterion, he or she would first assign to every conceivable probability distribution of the Earth temperature a numerical utility valuation - that may or may not have an expected utility form - and would compare alternative sets of probability distributions such as that of Figure 1 on the basis of their expected valuations under the assumption that all distributions in the set are equally likely. This uniform treatment of the different possible distributions of the Earth temperature is somewhat restrictive. Why would a public decision maker consider equally "credible" the different scientific studies that have given rise to the distributions of figure 1 ?

The criteria characterized in this paper avoid this limitation, while keeping the "smoothness" associated with the fact of evaluating a decision on the basis of some average value. Specifically, any criterion characterized herein can be thought of as resulting from the following two-step procedure. In the first step, the decision maker assigns to every conceivable distribution of the Earth temperature (say) two different numerical valuations. One such valuation is interpreted, just as in the UEU case, as reflecting the "utility" associated to the distribution. Again, this "utility" can, but does not need to, be an "expected utility". The other valuation, restricted by our characterization to be strictly positive, is interpreted as reflecting the a priori "plausibility" attached by the decision maker to every conceivable distribution of the Earth temperature. For instance, the decision maker may believe that a sure increase of the Earth tem-

\footnotetext{
${ }^{2}$ The median-based rankings characterized in Nitzan and Pattanaik (1984) are also subject to this difficulty although they avoid the criticism of been based on "extreme" values.
} 
perature by $3^{\circ}$ is more likely than the unpredictable distribution of that increase associated with some of the distributions of figure 1. In the second step, the decision maker compares alternative sets of distributions of the Earth temperature on the basis of their "expected utility", with expectations taken with respect to the likelihood function determined in the first step conditional on the fact that the distribution of Earth temperature is present in the set. We refer to any such criterion as to a Conditional Expected Utility (CEU) criterion. Any UEU criterion is a member of this family that assumes, in the first step, that all distributions of Earth temperature are equally likely. Hence the CEU family of criteria is a (significant) generalization of the UEU family that enables the decision maker to weights differently the different estimates of the distributions of the earth temperature in terms of their plausibility.

The CEU family of rankings of finite sets of objects characterized in this paper bears formal similarities with the family of criteria characterized in Ahn (2008) (and before him by Bolker (1966), Bolker (1967) and Jeffrey (1965)) for atomless sets of objects. A set of objects is atomless if, except perhaps for singleton sets (considered by Ahn (2008) but excluded by Bolker (1966), Bolker (1967) and Jeffrey (1965)), it always contains a proper subset that is not a singleton. Atomless sets contain therefore a continuum of elements and can not be finite like the set underlying figure 1, the urns considered in Ellsberg's experience or the choice of a medical treatment discussed in Ahn (2008). The fact that we consider only finite sets makes our setting very different one from that of Ahn (2008). As indicated in Gravel, Marchant, and Sen (2012), we believe that our finite subsets framework is an important one conceptually, at least from the viewpoint of practical implementability and testability, and descriptive faithfulness. We are not for instance aware of any public decision maker involved in regulating carbon emissions that would be given an atomless set of different probabilities distributions over the earth temperature.

The characterization of the CEU family of rankings of finite sets of objects obtained in this paper uses three axioms, and assumes that the objects are taken from a "rich" environment that may (or may not) be endowed with a topological structure. Two of our axioms are common with those Ahn (2008), and one of the two, called Averaging, was also used in the characterization of the UEU family of finite sets. Ahn (2008) obtains his characterization by combining the two axioms with two continuity conditions, and by exploiting the structure provided by his atomless set-theoretic structure. We obtain ours by combining the two axioms with an Archimedean condition, and by exploiting the assumed "richness" of the universe from which the finite sets are taken. Yet, and contrary to what we achieved for the characterization of UEU in Gravel, Marchant, and Sen (2012), we are not for the moment capable of providing a version of our main characterization result that would ride explicitly on a topological structure imposed on the universe of objects, and that would replace the richness condition and the archimedean axiom by an appropriate continuity condition. Moreover the richness condition that we use is, perhaps, unnecessarily strong. For one thing, it rules out, when applied to finite sets of objects taken from a topological space, any UEU criterion that uses a continuous utility function. 
The organization of the remaining of this paper is as follows. The next section introduces the formal setting and discusses the axioms and the family of criteria characterized. The results are presented in section 3, and discussed, along with examples showing the independence of the axioms, in Section 4. Section 5 provides some conclusion.

\section{The Model}

\section{$2.1 \quad$ Notation}

The sets of integers, non-negative integers, real numbers and non-negative real numbers are denoted respectively by $\mathbb{N}, \mathbb{N}_{+}, \mathbb{R}$ and $\mathbb{R}_{+}$. If $v$ is a vector in $\mathbb{R}^{k}$ for some strictly positive integer $k$ and $\alpha$ is a real number, we denote by $\alpha . v$ the scalar product of $\alpha$ and $v$. Our notation for vectors inequalities is $\geqq, \geq$ and $>$. By a binary relation $\succsim$ on a set $\Omega$, we mean a subset of $\Omega \times \Omega$. Following the convention in economics, we write $x \succsim y$ instead of $(x, y) \in R$. Given a binary relation $\succsim$, we define its symmetric factor $\sim$ by $x \sim y \Longleftrightarrow x \succsim y$ and $y \succsim x$ and its asymmetric factor $\succ$ by $x \succ y \Longleftrightarrow x \succsim y$ and not $(y \succsim x)$. A binary relation $\succsim$ on $\Omega$ is reflexive if the statement $x \succsim x$ holds for every $x$ in $\Omega$, is transitive if $x \succsim z$ always follows $x \succsim y$ and $y \succsim z$ for any $x, y, z \in \Omega$ and is complete if $x \succsim y$ or $y \succsim x$ holds for every distinct $x$ and $y$ in $\Omega$. An equivalence class $C$ of a binary relation $\succsim$ on $\Omega$ is a subset of $\Omega$ such that $c \sim c^{\prime}$ for all $c, c^{\prime} \in C$ and it is not the case that $c \sim c^{\prime}$ if $c \in C$ and $c^{\prime} \in \Omega \backslash C$. A reflexive, transitive and complete binary relation is called an ordering. An ordering is trivial if it has only one equivalence class.

\subsection{Basic concepts}

Let $X$ be an arbitrary universe of objects that we will refer to as "consequences". But keeping in mind the objective ambiguity context discussed in the preceding section, we could as well interpret $X$ as the set of all conceivable probability distributions over a more fundamental set of "prizes" (perhaps different rises of the average Earth temperature). While we do not make any specific assumptions on $X$, it will be clear subsequently that the axioms that we impose makes it natural to regard this set as infinite and rich.

We denote by $\mathcal{P}(X)$ the set of all non-empty finite subsets of $X$ (with generic elements $A, B, C$, etc.). Any such a subset is interpreted as a description of all consequences of an uncertain decision or, for short, as a decision. In an objective ambiguity setting, these consequences would be themselves probability distributions. A certain (non-ambiguous) decision with consequence $x \in X$ is identified by the singleton $\{x\}$.

Let $\succsim$ (with asymmetric and symmetric factors $\succ$ and $\sim$ respectively) be an ordering on $\mathcal{P}(X)$. We interpret the statement $A \succsim B$ as meaning "decision with consequences in $A$ is weakly preferred to decision with consequences in $B$ ". A similar interpretation is given to the statements $A \succ B$ ("strictly preferred 
to") and $A \sim B$ ("indifference").

We want to identify the properties (axioms) of the ordering $\succsim$ that are necessary and sufficient for the existence of a function $u: X \rightarrow \mathbb{R}$ and a function $p: X \rightarrow \mathbb{R}_{++}$that are such that that, for every $A$ and $B$ in $\mathcal{P}(X)$ :

$$
A \succsim B \Longleftrightarrow \frac{\sum_{a \in A} p(a) u(a)}{\sum_{a \in A} p(a)} \geq \frac{\sum_{b \in B} p(b) u(b)}{\sum_{b \in B} p(b)} .
$$

We refer to an ordering numerically represented as per (1) for some functions $p$ and $u$ as to a Conditional Expected Utility (CEU) criterion. Indeed, the function $p$ is naturally interpreted as assigning to every consequence - or lottery in the objective ambiguity framework - a number that reflects its a priori "plausibility", while the $u$ function is interpreted as a utility function that evaluates the "desirability" of every consequence from the decision maker's view point. Hence an ordering represented by (1) can be seen as comparing decisions on the basis of the expected utility of their consequences conditional upon the fact that they will materialize. We notice that the requirement that $p(x)>0$ for every $x \in X$ guarantees indeed that the "event" on which the conditioning is performed is well-defined.

We notice also that the family of UEU criteria characterized in Gravel, Marchant, and Sen (2012) is, a priori, a subclass of CEU family, in which the function $p$ is any constant function. Yet, as we shall see later, the characterization that we provide of this family is not complete as it does not cover all criteria that belong to the family represented by (1). The reason for this is that we characterize this family by assuming that both the universe $X$ and the ordering $\succsim$ satisfies the following "richness" condition (somewhat stronger than the condition of the same name used in Gravel, Marchant, and Sen (2012)).

Condition 1 Richness. For every sets $A, B, C, D$ and $D^{\prime}$ in $\mathcal{P}(X)$ such that $D^{\prime} \sim A \prec B \prec C \sim D$, there are sets $E$ and $E^{\prime}$ satisfying $E \cap(A \cup C \cup D)=$ $\varnothing=E^{\prime} \cap\left(A \cup C \cup D^{\prime}\right)$ such that $E \sim C, E^{\prime} \sim A$ and $E \cup A \sim B \sim E^{\prime} \cup C$.

This condition requires the domain to be sufficiently rich, and the ordering $\succsim$ to be sufficiently "smooth", for opening up the possibility of "matching" - in terms of indifference - any given decision by appropriate combinations of other decisions that are strictly better, and strictly worse than that decision. We emphasize that this condition restricts both the universe from which the objects are taken and the ordering $\succsim$. For instance, a "discontinuous" ordering like, say, the Leximin one that would compare sets on the basis of lexicographic extension of their "worst" - as per the ordering $\succsim$ restricted to singletons - elements would violate this condition. It is somewhat difficult to appraise the strength of this condition. On the one hand, it may seem to be a weak condition because its asserted existence of specific sets $E$ and $E^{\prime}$ is contingent upon the sets $A, B$, $C, D$ and $D^{\prime}$ having the properties indicated in the antecedent of the condition. On the other hand, as shown in the next section, the richness condition has 
some strength since, if $X$ is a topological space, it excludes from the class of rankings represented by (1) those for which the function $p$ is constant and continuous. Moreover, this richness condition is not necessary for an ordering to be represented by (1).

Before turning to the three axioms that are necessary (and sufficient) for an ordering on $\mathcal{P}(X)$ satisfying the richness condition to be a CEU criterion, we find useful to compare our framework to that of Ahn (2008), in which $X$ is explicitly taken to be the $k-1$ dimensional simplex $S^{k-1}:\left\{x \in \mathbb{R}_{+}^{k}: x_{j} \in[0,1]\right.$ for all $j=1, \ldots, k$ and $\left.\sum_{j=1}^{k} x_{j}=1\right\}$, interpreted as the set of all conceivable lotteries on some finite set of $k$ prices. Instead of considering finite subsets of $S^{k-1}$, Ahn (2008) applies his analysis to subsets of $S^{k-1}$ that are equal to the closure of their interior (using the topology of the Euclidean distance) and to singletons. This means that all non-singleton sets considered in Ahn (2008) contains continuously many elements. Ahn (2008) characterizes all orderings $\succsim$ of the subsets of $S^{k-1}$ that are equal to the closure of their interior - along with singletons - that can be written as:

$$
A \succsim B \Longleftrightarrow \frac{\int_{A} u(a) d \mu}{\mu(A)} \geq \frac{\int_{B} u(a) d \mu}{\mu(B)} .
$$

for some continuous function $u: S^{k-1} \longrightarrow \mathbb{R}$ and some probability measure $\mu$ on the Borel subsets of $S^{k-1}$. Orderings that can be represented as per (2) have also been characterized by Bolker (1966), Bolker (1967) and Jeffrey (1965) (see e.g. Broome (1990) for a nice discussion of the Bolker-Jeffrey theory). One can view the representation (1) as a finite version of the representation (2) in which the measure $\mu$ is defined, for any finite set $A$, by:

$$
\mu(A)=\sum_{a \in A} p(a)
$$

Yet we (over ?) emphasize that our restriction to finite sets makes the analysis very different from that of Ahn (2008) and Bolker (1966), Bolker (1967) and Jeffrey (1965).

The first axiom used in our characterization of the family of orderings of $\mathcal{P}(X)$ represented by $(1)$ is the following "Archimedean" one.

Axiom 1 Archimedean. For all sets $A, B, C, D$ and $E$ in $\mathcal{P}(X)$ such that $A \sim B \sim C \sim D \nsim E, A \cup E \succ B \cup E$ and $E \cap(C \cup D)=\emptyset$, if there are two infinite sequences of sets $A_{0}, A_{1}, \ldots, A_{i}, \ldots$ and $B_{0}, B_{1}, \ldots, B_{i}, \ldots$, satisfying $A_{i} \cap\left(E \cup C \cup A_{j}\right)=\emptyset, B_{i} \cap\left(E \cup D \cup B_{j}\right)=\emptyset, A_{i} \sim A, B_{i} \sim B, A_{i} \cup E \sim A \cup E$ and $B_{i} \cup E \sim B \cup E$ for all $i \neq j \in \mathbb{N}$, then there must be some $n \in \mathbb{N}$ for which $C \cup E \bigcup_{i=0}^{n} A_{i} \succsim D \cup E \bigcup_{i=0}^{n} B_{i}$ holds .

As usual, Archimedean axioms are difficult to write but they say a simple thing: no decision is infinitely more valuable than any other. As stated here, 
the axiom applies to decisions $A$ and $B$ between which the decision maker is indifferent. Suppose that the uncertainty surrounding these decisions is increased in the sense that the set of their possible consequences is "enlarged" to consequences in some set $E$ that are not equivalent to $A$ and $B$. Perhaps $E$ is a set of consequences that the decision maker considers better than $A$ or $B$. Perhaps it is worse. Suppose also that the decision that leads to consequences in $A \cup E$ is strictly better than a decision with consequences in $B \cup E$. Consider then replacing, in this enlargement to $E$, the initial sets of consequences $A$ and $B$ by any set in some sequences $A_{j}$ and $B_{j}$ (respectively, for $j=0, \ldots$,) that are, again respectively, disjoint from $A$ and from $B$. These sets are also, in every sequence, disjoint from each other. Suppose that this replacement is a matter of indifference for the decision maker. Intuitively then, the sets $A_{0}, A_{1}, \ldots, A_{i}, \ldots$ can all be considered to be "clones" of $A$ relative to $E$ in the sense that the decision maker is totally indifferent between a decision with consequences in any of these sets or in $E$ and a decision with consequences in $A$ or in $E$. Similarly, sets $B_{0}, B_{1}, \ldots, B_{i}, \ldots$ are clones of $B$ relative to $E$. The Archimedean axiom says that replacing, in this enlargement to $E$, decision $A$ by an equivalent decision $C$ and replacing, in the very same enlargement to $E$, decision $B$ by an equivalent decision $C$ can not reverse the ranking of $C \cup E$ vis-à-vis $D \cup E$ to such an extent that the reversal - if any - can not be outweighed by adding to $C \cup E$ and to $D \cup E$ a suitably long sequence of clones of $A$ and $B$ respectively. That is, decisions $C$ and $D$ can not be "infinitely more important" than decisions $A$ and $B$ relative to $E$ when they are themselves indifferent to $A$ and $B$ respectively.

While this axiom may seem technical and, when understood, "natural", it is required in the characterization, as shown in example 1 of section 4. Ahn (2008) does not use an Archimedean axiom. He uses, instead, two continuity axioms that can not be defined in the abstract universe considered here that may not have a topological structure.

The two next axioms however are used by Ahn. The first of them is the Averaging axiom (using the terminology of Broome (1990)) that was also used in the characterization of the UEU family of criteria provided in Gravel, Marchant, and Sen (2012). The formal statement of this axiom is as follows.

Axiom 2 Averaging. Suppose $A$ and $B \in \mathcal{P}(X)$ are disjoint. Then $A \succsim B$ iff $A \cup B \succsim B$ iff $A \succsim A \cup B$.

This axiom was called "disjoint set betweenness" by Ahn (2008). It says that enlarging the possible outcomes of a decision $A$ to those of a (disjoint) decision $B$ is worth doing (resp. not worth doing) if and only if the set $B$ of added consequence is better (resp. worse) than the set $A$ to which it is added. It captures an intuitive property satisfied by calculations of "average" in various settings (e.g. adding a student to a class will increase the average of the class if and only if the grade of the added student is larger than the average of the class). The "only if" part of the axiom is strong since it asserts that the only reason for ranking a set $B$ above (resp. below) a set $A$ is when the addition of $B$ to $A$ is considered a good (resp. bad) thing. A weaker version of Averaging 
(that only requires the "if" part in its statement) is used in Olszewski (2007). A very similar axiom is also used in Gul and Pesendorfer (2001) for ranking menus of alternatives in a way that reflects "temptation" and "self-control".

The last axiom is called Balancedness by Ahn (2008). It is stated as follows.

Axiom 3 Balancedness. Suppose $A$ and $B$ are two sets in $\mathcal{P}(X)$ such that $A \sim B$. Then, if there is a set $C \in \mathcal{P}(X)$ satisfying $(A \cup B) \cap C=\varnothing$ and $A \sim B \succ C$ for which $A \cup C \succsim B \cup C$ holds, $A \cup D \succsim B \cup D$ must hold for all sets $D \in \mathcal{P}(X)$ for which $(A \cup B) \cap D=\varnothing$ and $A \sim B \succ D$.

This axiom is a separability condition that plays a key role in guaranteeing that the measure of finite sets provided (as per expression (3)) by the function $p$ in the representation (1) is well-defined. The difficulty indeed in the characterization of the family of CEU criteria is to disentangle the role played by the two functions of expression (1) that represent two different notions. The function $u$ serves as identifying the "utility" of a decision. The function $p$ serves as identifying the likelihood of the outcomes of the decision. When do we have evidence that a (finite) collection of outcomes of a decision is "more likely" than another ? One such evidence - put forth by the balancedness axiom - is provided when two decisions $A$ and $B$ are equivalent for the decision maker in utility terms, but are not anymore equivalent if the outcomes that they may yield are enlarged to outcomes of another decision $C$ that is considered worse to both $A$ and $B$. Suppose specifically that a decision leading to either $A$ or $C$ is better than a decision leading to either $B$ or $C$. Such a preference for $A \cup C$ over $B \cup C$ can only come from the fact that the good outcomes in $A \cup C$ (that are in $A$ ) are "more likely" than the good outcomes (in $B$ ) in $B \cup C$. The balancedness axiom guarantees that the definition of what it means for $A$ to be more likely than $B$ does not depend upon which particular set $C$ worse than both $A$ and $B$ is chosen.

\section{Main results}

Let us define the sets $m(X)$ and $M(X)$ of minimal (resp. maximal) decisions in $X$ by $m(X)=\{A \in \mathcal{P}(X): A \precsim B \forall B \in \mathcal{P}(X)\}$ and $M(X)=\{A \in \mathcal{P}(X)$ : $A \succsim B \forall B \in \mathcal{P}(X)\}$. Each of these set can of course be empty. We define the set $\mathcal{P}_{*}(X)$ by $\mathcal{P}_{*}(X)=\mathcal{P}(X) \backslash(m(X) \cup M(X))$. Hence, the set $\mathcal{P}_{*}(X)$ contains all finite subsets of $X$ that are not maximal or minimal with respect to the ordering $\succsim$. One may of course have $\mathcal{P}_{*}(X)=\mathcal{P}(X)$ if there are no maximal nor minimal sets for the ordering $\succsim$. Yet, we know of at least one context where the set $\mathcal{P}_{*}(X)$ will be different from $\mathcal{P}(X)$. This will be the case if, as in Ahn (2008), the universe $X$ is the $k-1$ dimensional simplex interpreted as the set of all lotteries on a finite set of prices. In such a setting, it would seem natural that there be a "best" prize (say the certainty that no increase in the Earth temperature will take place in the next 50 years) and a "worst prize" (say the certainty that the earth temperature will increase by $10^{\circ} \mathrm{C}$ in the next 50 years). If this is the case, the singleton that gives unambiguously the lottery that assigns 
a probability 1 to the best (resp. to the worst) prize would be maximal (resp. minimal) in $\mathcal{P}(X)$.

We first prove the result on the set $\mathcal{P}_{*}(X)$. Once having obtained the numerical representation as per $(1)$ on $\mathcal{P}_{*}(X)$, we show that the representation can be extended to the whole set $\mathcal{P}(X)$. The proof is based on several auxiliary results that we now present. We relegate all proofs in the Appendix.

The first result is the simple, but important, fact that if the ordering $\succsim$ is not trivial and satisfies Averaging, then if decisions $A$ and $B$ are respectively maximal and minimal in the set $\mathcal{P}(X)$, then $A$ and $B$ must be disjoint. We formally state this result as follows.

Lemma 1 Let $\succsim$ be a non-trivial ordering of $\mathcal{P}(X)$ satisfying Averaging. Then if sets $A$ and $C \in \mathcal{P}(X)$ are such that $A \succsim B \succsim C$ for all sets $B \in \mathcal{P}(X)$, then $A \cap C=\varnothing$.

The second result establishes a somewhat strong implication of the Richness condition when it is combined with the Averaging axiom, and applied to a nontrivial ordering. Indeed, the richness condition implies that, for any two decisions faced by the decision maker, is possible to replace one them by another that is indifferent to it and that leads to different consequences than those of the two initial decisions. The formal statement of this lemma is as follows.

Lemma 2 Let $\succsim$ be a non-trivial ordering of $\mathcal{P}(X)$ satisfying Richness and Averaging. Then, for every $A, C \in \mathcal{P}(X)$, there exists $B \in \mathcal{P}(X)$ such that $B \sim A$ and $B \cap(A \cup C)=\varnothing$.

An important implication of this lemma, and of the richness condition on which it rides, is that any ordering of $\mathcal{P}(X)$ satisfying Averaging and Richness if the universe $X$ is finite must be trivial. Averaging and Richness, if they are to apply to a non-trivial ordering, force $X$ to be infinite. More precisely, it forces the set $\mathcal{P}_{*}(X)$ to be itself infinite in the sense that, for any decision $B \in \mathcal{P}_{*}(X)$, one can find decisions $A$ and $C$ in $\mathcal{P}_{*}(X)$ that are, respectively, strictly better and strictly worse than $B$. Hence, the set $\mathcal{P}_{*}(X)$ of non-maximal and non-minimal decisions is not only infinite. It is also "unbounded" with respect to the ordering $\succsim$. The formal statement of this fact is as follows.

Lemma 3 If $\succsim$ is a non-trivial ordering on $\mathcal{P}(X)$ satisfying Richness and $A v$ eraging, then, for every set $B \in \mathcal{P}_{*}(X)$, there are decisions $A$ and $C \in \mathcal{P}_{*}(X)$ such that $A \prec B \prec C$.

Endowed with these two first lemma, we define, for any decision $E \in \mathcal{P}_{*}(X)$, the set $\mathcal{P}^{E}(X)=\{C \in \mathcal{P}(X): C \sim E\}$ of all decisions that are equivalent to $E$. This set is not empty since it contains $E$ itself by reflexivity. We then define the binary relation $\succsim_{l}$ on $\mathcal{P}^{E}(X)$ by: $A \succsim_{l} B$ iff there exists a decision $C$ disjoint from $A$ and $B$ such that $A \cup C \succsim B \cup C$ and $C \prec E$. Notice that, since we work on the set $\mathcal{P}_{*}(X)$, we do not define $\succsim_{l}$ on a maximal (or minimal) equivalence class. This binary relation $\succsim_{l}$ is naturally interpreted as meaning "is at least as 
probable as". Hence decision $A$ is at least as probable as decision $B$ if $A$ and $B$ provides the decision maker with the same "utility" - equal to that of the benchmark decision $E$ - and if merging $A$ to a strictly worse decision $C$ is better than merging $B$ with that same worse decision. Thanks to the Balancedness axiom, this binary relation is well-defined in the sense that it des not depend upon the particular set $C$ used to define it. The following lemma, also proved in the Appendix, establishes more precisely that the binary relation $\succsim_{l}$ is in fact an ordering of the set $\mathcal{P}^{E}(X)$.

Lemma 4 Assume that $\succsim$ is a non-trivial ordering on $\mathcal{P}(X)$ satisfying Balancedness and Averaging. Then, for any decision $E \in \mathcal{P}_{*}(X)$, the relation $\succsim_{l}$ is an ordering of $\mathcal{P}^{E}(X)$.

In the next lemma, we establish the formal definition of the asymmetric factor $\succ^{l}$ and the symmetric factor $\sim^{l}$ of the ordering $\succsim^{l}$.

Lemma 5 Assume that $\succsim$ is an ordering on $\mathcal{P}(X)$ satisfying Richness, Balancedness and Averaging. Then, for any decision $E \in \mathcal{P}_{*}(X)$, any decisions $A$ and $B$ in $\mathcal{P}^{E}(X)$ and any decision $C \in \mathcal{P}(X)$ such that $E \succ C$ and $C \cap(A \cup B)=$ $\varnothing$,

$$
\begin{aligned}
& \text { 1. } A \succ_{l} B \text { if and only if } A \cup C \succ B \cup C \text {. } \\
& \text { 2. } A \sim_{l} B \text { if and only if } A \cup C \sim B \cup C \text {. }
\end{aligned}
$$

The next lemma is quite important. It establishes the possibility of representing the "plausibility" ordering $\succsim^{l}$ of sets that are indifferent to each other - as per the ordering $\succsim$ - by a set-additive strictly positive numerical function which behaves indeed like a probability measure. The proof this lemma rides on an important theorem on additive numerical representation established in Krantz, Luce, Suppes, and Tversky (1971).

Lemma 6 Assume that $\succsim$ is a non-trivial ordering on $\mathcal{P}(X)$ satisfying Richness, Balancedness, Averaging and the Archimedean axiom. Then, for every decision $E \in \mathcal{P}_{*}(X)$, there exists a mapping $p^{E}: \mathcal{P}^{E}(X) \rightarrow \mathbb{R}_{++}$such that, for all $A, B \in \mathcal{P}^{E}(X), A \succsim_{l} B$ iff $p^{E}(A) \geq p^{E}(B)$ and, for all disjoint $A, B \in \mathcal{P}^{E}(X)$, $p^{E}(A \cup B)=p^{E}(A)+p^{E}(B)$. Furthermore, $p^{E}$ is unique up to a linear transformation.

Given any decision $E$, the ordering $\succsim^{l}$ and its additive numerical representation $p^{E}$ enables the comparison of any two decisions that are indifferent to $E$ as per the ordering $\succsim$. We now need to establish how the binary relation $\succsim^{l}$ compares - in terms of plausibility - decisions that are not indifferent to each other in terms of the ordering $\succsim$. A preliminary step for doing so consists in showing the possibility of constructing, starting from $p^{E}$, an additively separable function which, for any decision $E$, indicates whether any other decision is weakly preferred to $E$, or weakly worse than $E$. We do that in the following 
lemma, that is very similar in its statement and proof as Lemma 10 in Ahn (2008).

Lemma 7 Assume that $\succsim$ is a non-trivial ordering on $\mathcal{P}(X)$ satisfying Richness, Balancedness, Averaging and the Archimedean axiom. If $E \in \mathcal{P}_{*}(X)$, there exists a mapping $\nu^{E}: \mathcal{P}(X) \rightarrow \mathbb{R}$ such that (i) $A \cap B=\varnothing$ implies $\nu^{E}(A \cup B)=\nu^{E}(A)+\nu^{E}(B)$ and $($ ii $) \nu^{E}(A) \geq 0$ iff $A \succsim E$ and $\nu^{E}(A) \leq 0$ iff $A \precsim E$.

The function $\nu^{E}$ constructed in the proof of Lemma 7 is a somewhat complex - but yet additively separable - extension of the function $p^{E}$ of Lemma 6 . An important thing to notice about the numerical function $\nu^{E}$ is that, while defined only with respect to a decision $E \in \mathcal{P}_{*}(X)$, it is in fact a function that maps every decision $A \in \mathcal{P}(X)$ into the set of real number. Hence, the domain of $\nu^{E}$ includes sets that belong to $m(X)$ or $M(X)$.

The additively separable function $\nu^{E}$ of Lemma 7 enables one to identify whether some decision is better or worse than the benchmark decision $E$. In order to obtain a numerical representation of the whole preference $\succsim$ over all sets, it is important to connect together the information conveyed by the functions $\nu^{E}$ for all benchmark decisions $E$. A first step in establishing this connection is the following lemma, which says that the functions $\nu^{A}$, defined with respect to some reference decision $A$, can actually be used to numerically represent the plausibility ordering $\succsim^{l}$ defined on the set $\mathcal{P}^{B}(X)$ of all decisions that are equivalent - as per the ordering $\succsim$ - to a decision $B$ that is not itself equivalent to $A$. Put differently, the function $\nu^{A}$ numerically represents the plausibility ordering $\succsim^{l}$ defined on $\mathcal{P}^{B}(X)$ no matter what is the reference set $B$. The formal statement of this result is as follows.

Lemma 8 Assume that $\succsim$ is a non-trivial ordering on $\mathcal{P}(X)$ satisfying Richness, Balancedness, Averaging and the Archimedean axiom. Then, for any two sets $A, B \in \mathcal{P}_{*}(X)$ the function $\nu^{B}$ numerically represents the plausibility ordering $\succsim^{l}$ on $\mathcal{P}^{A}(X)$ in the sense that, for any two decisions $S$ and $T \in \mathcal{P}^{A}(X)$ $S \succsim^{l} T \Longleftrightarrow \nu^{B}(S) \geq \nu^{B}(T)$.

We now establish, with the help of this result, that the set of all functions $\nu^{E}$ obtained for all reference decisions $\left.E \in \mathcal{P}_{*}(X)\right)$ is a "two-dimensional space" in the sense that any such function can be obtained as a linear combination of any two other linearly independent functions. A somewhat analogous result was proved as Lemma A12 in Ahn (2008).

Lemma 9 Assume that $\succsim$ is a non-trivial ordering on $\mathcal{P}(X)$ satisfying Richness, Balancedness, Averaging and the Archimedean axiom. Then the family $\left\{\nu^{E}: E \in \mathcal{P}_{*}(X)\right\}$ is spanned by any two of its members $\nu^{A}$ and $\nu^{B}$ provided that $\nu^{A}$ and $\nu^{B}$ are linearly independent). That is, for any two functions $\nu^{A}$ and $\nu^{B}$ for which there are is no real number $\alpha$ such that $\frac{\nu^{A}(C)}{\nu^{B}(C)}=\alpha$ for all decisions $C \in \mathcal{P}(X)$, one can write any function $\nu^{E}$ as $\nu^{E}=\alpha^{E} \nu^{A}+\beta^{E} \nu^{B}$ for some real numbers $\alpha^{E}$ and $\beta^{E}$. 
The next lemma establishes a somewhat stronger result concerning the set of functions $\left\{\nu^{E}: E \in \mathcal{P}_{*}(X)\right\}$ defined in Lemma 7. Namely, that this set is a positive cone.

Lemma 10 Assume that $\succsim$ is a non-trivial ordering on $\mathcal{P}(X)$ satisfying Richness, Balancedness, Averaging and the Archimedean axiom. Let $A, B$ and $C$ be three sets in $\mathcal{P}_{*}(X)$. Then, there does not exist a strictly positive real number $\lambda$ and $a \delta \in[0,1]$ such that $-\lambda \nu^{A}(D)=\delta \nu^{B}(D)+(1-\delta) \nu^{C}(D)$ holds for all set $D \in \mathcal{P}(X)$.

Using these results on the (vector-like) structure of the set of functions $\left\{\nu^{E}\right.$ : $\left.E \in \mathcal{P}_{*}(X)\right\}$ defined in Lemma 7 , we now use these functions to construct a disjoint-set additive function $\mu$ that will play a key role in the numerical representation of the form (1) that we are aiming at. Roughly speaking, the function $\mu$ will define the "denominator" of the numerical expression (1).

Lemma 11 Assume that $\succsim$ is a non-trivial ordering on $\mathcal{P}(X)$ satisfying Richness, Balancedness, Averaging and the Archimedean axiom. Then there exists a disjoint-additive mapping $\mu: \mathcal{P}(X) \rightarrow \mathbb{R}$ such that $\mu(C)>0$ for all $C \in \mathcal{P}_{*}(X)$ and such that $\mu$.

The next lemma establishes that the set function $f^{C}: \mathcal{P}_{*}(X) \rightarrow \mathbb{R}$ defined, for any reference set $C$, by:

$$
f^{C}(A)=\frac{v^{C}(A)}{\mu(A)}
$$

provides a numerical representation of the ordering $\succsim$ on the set $\mathcal{P}_{*}(X)$.

Lemma 12 Assume that $\succsim$ is a non-trivial ordering on $\mathcal{P}(X)$ satisfying Richness, Balancedness, Averaging and the Archimedean axiom. Choose any $C \in$ $\mathcal{P}_{*}(X)$. Then, for all sets $A$ and $B \in \mathcal{P}_{*}(X), \nu^{C}(A) / \mu(A) \geq \nu^{C}(B) / \mu(B)$ iff $A \succsim B$.

In the next lemma, we show that each of the two disjoint set-additive functions $\nu^{C}$ - for any set $C \in \mathcal{P}_{*}(X)$ - and $\mu$ serves as an index of the equivalence class associated to the intersection of the symmetric factors of the two orderings $\succsim$ and $\succsim^{l}$. That is, any two sets of consequences that are considered both equally desirable - from the view point of $\succsim$ - and equally "plausible" - as per $\succsim^{l}$ - will be assigned the same value by either the function $\nu^{C}$ or the function $\mu$.

Lemma 13 Assume that $\succsim$ is a non-trivial ordering on $\mathcal{P}(X)$ satisfying Richness, Balancedness, Averaging and the Archimedean axiom. Then, for any $C \in$ $\mathcal{P}_{*}(X)$, and any two decisions $A$ and $B \in \mathcal{P}_{*}(X), A \sim B$ and $A \sim_{l} B$ implies $\nu^{C}(A)=\nu^{C}(B)$ and $\mu(A)=\mu(B)$.

We now establish the existence, in the universe $X$, of consequences that have "arbitrarily small" level of plausibility. More precisely, we show that the 
function $\mu$ that defines the denominator of the numerical expression (1) can take values arbitrarily close to zero if the set of consequences to which it applies is suitably chosen. Notice that this implies that the UEU criteria characterized in Gravel, Marchant, and Sen (2012) are not members of the family of CEU criteria that are represented as per (1) for some functions $p$ and $u$ (with $p$ strictly positive). Indeed, if a UEU criterion was a CEU criterion, the function $p$ of expression (1) would be a constant (say $p(x)=a$ for some strictly positive number $a$ for all consequences $x$ ). In this case, there would be no consequences in $X$ with "arbitrarily small" level of plausibility. The contribution of the richness condition to this fact that 0 is the greatest lower bound of the the function $\mu$ is very important.

Lemma 14 Assume that $\succsim$ is a non-trivial ordering on $\mathcal{P}(X)$ satisfying Richness, Balancedness, Averaging and the Archimedean axiom. Then, for any set $D \in \mathcal{P}_{*}(X)$ and any strictly positive real number $\varepsilon$, one can find a decision $E$ such that $E \sim D$ and $\mu(E)<\epsilon$.

The results obtained so far have been dealing with decisions that are not maximal or minimal - for the ordering $\succsim$ - in the set $\mathcal{P}(X)$. We must now progress in showing that the functions $\mu$ and $\nu^{C}$ (for any given $C \in \mathcal{P}_{*}(X)$ ) defined for those non-minimal or maximal decisions can also be extended to minimal or maximal decisions (if any). The first step in this direction is accomplished in the next lemma, that extends the function $\mu$ of Lemma 11 - that was taking strictly positive value on all sets in $\mathcal{P}_{*}(X)$ - to a closely related function $\mu_{+}$ which takes strictly positive value on every set in $\mathcal{P}(X)$ (including therefore maximal and/or minimal sets in $\mathcal{P}(X)$, if any).

Lemma 15 Assume that $\succsim$ is a non-trivial ordering on $\mathcal{P}(X)$ satisfying Richness, Balancedness, Averaging and the Archimedean axiom. There exist then a disjoint-additive mapping $\mu_{+}: \mathcal{P}(X) \rightarrow \mathbb{R}$ such that $\mu_{+}(S)>0$ for all $S \in \mathcal{P}(X)$. Moreover the function $\mu_{+}$belongs to the family $\left\{\nu^{E}: E \in \mathcal{P}_{*}(X)\right\}$ spanned by any two of its linearly independent members $\nu^{A}$ and $\nu^{B}$.

Endowed with this function, we need now to prove an analogue of Lemma 12, but using $\mu_{+}$rather than $\mu$. We do this in the following lemma.

Lemma 16 Assume that $\succsim$ is a non-trivial ordering on $\mathcal{P}(X)$ satisfying Richness, Balancedness, Averaging and the Archimedean axiom. Choose any $C \in$ $\mathcal{P}_{*}(X)$. Then, for all sets $A$ and $B \in \mathcal{P}(X), \nu^{C}(A) / \mu_{+}(A) \geq \nu^{C}(B) / \mu_{+}(B)$ iff $A \succsim B$. Choose any $C \in \mathcal{P}_{*}(X)$ and define $\nu=\nu^{C}$. Then, for all $A, B \in \mathcal{P}_{*}(X)$, $\nu(A) / \mu_{+}(A) \geq \nu(B) / \mu_{+}(B)$ iff $A \succsim B$.

We have now gather all the auxiliary results that are required to prove our main theorem, that is as follows.

Theorem 1 Assume that $\succsim$ is an ordering of $\mathcal{P}(X)$ that satisfies Richness. Then $\succsim$ satisfies Balancedness, Averaging and the Archimedean axiom if and only if there are two functions $u: X \rightarrow \mathbb{R}$ and $p: X \rightarrow \mathbb{R}_{++}$such that (1) holds. 


\section{Interpretation of the results}

\subsection{Independence of the axioms}

In the next three examples, we show that the axioms used in the characterization of the CEU family of orderings are independent when applied to an ordering satisfying richness. The first example exhibits an ordering of $\mathcal{P}(X)$ that does not belong to the CEU family but that satisfies averaging, balancedness and richness (but not the Archimedean axiom).

Example 1 Let $X=\mathbb{R}_{++}^{2} \times \mathbb{R}^{2}$. For every $A \in \mathcal{P}(X)$, define

$$
U_{1}(A)=\frac{\sum_{a \in A} a_{1} a_{3}}{\sum_{a \in A} a_{1}}
$$

and

$$
U_{2}(A)=\frac{\sum_{a \in A} a_{2} a_{3}}{\sum_{a \in A} a_{2}} .
$$

Define $\succsim$ on $\mathcal{P}(X)$ by.

$$
\begin{aligned}
& A \sim B \quad \Longleftrightarrow U_{1}(A)=U_{1}(B) \text { and } U_{2}(A)=U_{2}(B) ; \\
& A \succ B \\
& \begin{array}{l}
U_{1}(A)>U_{1}(B) \\
\text { or } \\
U_{1}(A)=U_{1}(B) \text { and } U_{2}(A)>U_{2}(B) .
\end{array}
\end{aligned}
$$

We first show that this ranking violates the Archimedean axiom. Let $A=$ $\{(1,2,0,-1)\}, B=\{(1,1,0,-1)\}, A_{i}=\{(1,2,0, i)\}, B_{i}=\{(1,1,0, i)\}, C=$ $\{(1,1,0,0)\}$ and $D=\{(2,1,0,0)\}$. We clearly have $A \sim A_{i} \sim B \sim B_{i} \sim C \sim D$ for all $i \in \mathbb{N}$. Let $F=\{(0,0,-1,0)\}$. We have $A \succ F, A \cup F \succ B \cup F$, $A_{i} \cup F \sim A \cup F$ and $B_{i} \cup F \sim B \cup F$ for all $i \in \mathbb{N}$. Yet, contrary to what the Archimedean axiom requires, $C \cup F \bigcup_{i=0}^{n} A_{i} \prec D \cup F \bigcup_{i=0}^{n} B_{i}$ for all $n \in \mathbb{N}$.

We next show that $\succsim$ satisfies Averaging. Suppose first that $A \succ B$. Using the definition of $\succsim$, this is either equivalent to:

$$
\begin{aligned}
U_{1}(A) & >U_{1}(B) \\
& \Longleftrightarrow \\
U_{1}(A) & >U_{1}(A \cup B)>U_{1}(B) \\
& \Longleftrightarrow \\
A & \succ A \cup B \succ B
\end{aligned}
$$

or to: 


$$
\begin{aligned}
U_{1}(A) & =U_{1}(B) \text { and } U_{2}(A)>U_{2}(B) \\
& \Longleftrightarrow \\
U_{1}(A) & =U_{1}(A \cup B)=U_{1}(B) \text { and } U_{2}(A)>U_{2}(A \cup B)>U_{2}(B) \\
& \Longleftrightarrow \\
A & \succ A \cup B \succ B .
\end{aligned}
$$

$A$ similar reasoning holds when $A \sim B$. To show that $\succsim$ satisfies Richness, consider $A, B, C \in \mathcal{P}(X)$ such that $A \succ B \succ C$. We will show that there exists a set $D=\{d, e\}$ such that $D \cap(A \cup C)=\emptyset, D \sim A$ and $D \cup C \sim B$. So, we must have

$$
\begin{gathered}
\frac{d_{1} d_{3}+e_{1} e_{3}}{d_{1}+e_{1}}=U_{1}(A), \\
\frac{d_{2} d_{3}+e_{2} e_{3}}{d_{2}+e_{2}}=U_{2}(A), \\
\frac{d_{1} d_{3}+e_{1} e_{3}+\sum_{c \in C} c_{1} c_{3}}{d_{1}+e_{1}+\sum_{c \in C} c_{1}}=U_{1}(B), \\
\frac{d_{2} d_{3}+e_{2} e_{3}+\sum_{c \in C} c_{2} c_{3}}{d_{2}+e_{2}+\sum_{c \in C} c_{2}}=U_{2}(B) .
\end{gathered}
$$

Set $d_{3}=\max \left(U_{1}(A), U_{2}(A)\right)+1$ and $e_{3}=\min \left(U_{1}(A), U_{2}(A)\right)-1$. There clearly exist $d_{1}, e_{1} \in \mathbb{R}_{++}$such that (5) holds. Notice that $d_{1}, e_{1}$ are not unique; they can be scaled by any positive constant and we can choose this constant so that (7) holds. Similarly, there clearly exist $d_{2}, e_{2} \in \mathbb{R}_{++}$such that (6) holds. They are unique up to a multiplication by a positive constant, that we can choose independently of the scaling constant for $d_{1}, e_{1}$. So, we can choose it so that (8) holds. In order to guarantee that $D \cap(A \cup C)=\emptyset$, we can freely manipulate $d_{4}$ and $e_{4}$. Hence Richness holds. Finally, to show that $\succsim$ satisfies Balancedness, consider finite and non-empty subsets $A, B, C, D$ of $X$ such that $A \sim B \succ C, D$ and $(A \cup B) \cap(C \cup D)=\emptyset$. We have $A \cup C \succsim B \cup C$ if and only if either: $U_{1}(A \cup C)>U_{1}(B \cup C)$ iff $U_{1}(A \cup D)>U_{1}(B \cup D)$ iff $A \cup D \succsim B \cup D$ or $\left[U_{1}(A \cup C)=U_{1}(B \cup C)\right.$ and $\left.U_{2}(A \cup C) \geq U_{2}(B \cup C)\right] \quad$ iff $\left[U_{1}(A \cup D)=\right.$ $U_{1}(B \cup D)$ and $\left.U_{2}(A \cup D) \geq U_{2}(B \cup D)\right]$ iff $A \cup D \succsim B \cup D$.

The next example, provides a non-CEU ordering that satisfies balancedness, richness and the Archimedean axiom but violates averaging.

Example 2 Let $X=\mathbb{R}_{++} \times \mathbb{R}^{2}, p(x)=x_{1}, u(x)=x_{2}$,

$$
U(A)=\frac{\sum_{a \in A} p(a) u(a)}{\left(\sum_{a \in A} p(a)\right)^{2}}
$$

and $A \succsim B$ iff $U(A) \geq U(B)$.

The ranking $\succsim$ clearly satisfies Richness and the Archimedean axiom. It violates 
Averaging because $A=\{(3 / 4,2,0)\} \sim B=\{(3 / 4,2,1)\} \succ A \cup B$.

Let us prove that $\succsim$ satisfies Balancedness. $A \sim B$ implies:

$$
\sum_{a \in A} p(a) u(a)\left(\sum_{b \in B} p(b)\right)^{2}=\left(\sum_{a \in A} p(a)\right)^{2} \sum_{b \in B} p(b) u(b) .
$$

while $A \cup C \succsim B \cup C$ implies:

$$
\begin{aligned}
& \left(\sum_{a \in A} p(a) u(a)+\sum_{c \in C} p(c) u(c)\right)\left(\left(\sum_{b \in B} p(b)\right)^{2}+\left(\sum_{c \in C} p(c)\right)^{2}\right) \\
\geq & \left(\sum_{b \in B} p(b) u(b)+\sum_{c \in C} p(c) u(c)\right)\left(\left(\sum_{a \in A} p(a)\right)^{2}+\left(\sum_{c \in C} p(c)\right)^{2}\right)
\end{aligned}
$$

or, after distributing:

$$
\begin{aligned}
& \sum_{a \in A} p(a) u(a)\left(\sum_{b \in B} p(b)\right)^{2}+\sum_{c \in C} p(c) u(c)\left(\sum_{b \in B} p(b)\right)^{2}+\sum_{a \in A} p(a) u(a)\left(\sum_{c \in C} p(c)\right)^{2} . \\
& \geq \sum_{b \in B} p(b) u(b)\left(\sum_{a \in A} p(a)\right)^{2}+\sum_{c \in C} p(c) u(c)\left(\sum_{a \in A} p(a)\right)^{2}+\sum_{b \in B} p(b) u(b)\left(\sum_{c \in C} p(c)\right)^{2}
\end{aligned}
$$

Substituting (9) into this equation yields:

$$
\begin{aligned}
& \sum_{c \in C} p(c) u(c)\left(\sum_{b \in B} p(b)\right)^{2}+\sum_{a \in A} p(a) u(a)\left(\sum_{c \in C} p(c)\right)^{2} \\
& \geq \sum_{c \in C} p(c) u(c)\left(\sum_{a \in A} p(a)\right)^{2}+\sum_{b \in B} p(b) u(b)\left(\sum_{c \in C} p(c)\right)^{2}
\end{aligned}
$$

or:

$\sum_{c \in C} p(c) u(c)\left(\left(\sum_{b \in B} p(b)\right)^{2}-\left(\sum_{a \in A} p(a)\right)^{2}\right) \geq\left(\sum_{b \in B} p(b) u(b)-\sum_{a \in A} p(a) u(a)\right)\left(\sum_{c \in C} p(c)\right)^{2}$.

Since $\left(\sum_{c \in C} p(c)\right)^{2}>0$, one obtains:

$$
\begin{aligned}
& \frac{\sum_{c \in C} p(c) u(c)}{\left(\sum_{c \in C} p(c)\right)^{2}} \geq \frac{\sum_{b \in B} p(b) u(b)-\sum_{a \in A} p(a) u(a)}{\left(\sum_{b \in B} p(b)\right)^{2}-\left(\sum_{a \in A} p(a)\right)^{2}}=\frac{\sum_{b \in B} p(b) u(b)}{\left(\sum_{b \in B} p(b)\right)^{2}} \\
& \text { if } \quad\left(\sum_{b \in B} p(b)\right)^{2}-\left(\sum_{a \in A} p(a)\right)^{2}>0 \text { or } \\
& \quad \frac{\sum_{c \in C} p(c) u(c)}{\left(\sum_{c \in C} p(c)\right)^{2}} \leq \frac{\sum_{b \in B} p(b) u(b)-\sum_{a \in A} p(a) u(a)}{\left(\sum_{b \in B} p(b)\right)^{2}-\left(\sum_{a \in A} p(a)\right)^{2}}=\frac{\sum_{b \in B} p(b) u(b)}{\left(\sum_{b \in B} p(b)\right)^{2}}
\end{aligned}
$$


if $\left(\sum_{b \in B} p(b)\right)^{2}-\left(\sum_{a \in A} p(a)\right)^{2}<0$. Inequality (10) is not possible because $B \succ$ $C$. We therefore conclude that Inequality (11) holds and that $\left(\sum_{b \in B} p(b)\right)^{2}-$ $\left(\sum_{a \in A} p(a)\right)^{2} \leq 0$.

We also know that $D \prec B$. This implies:

$$
\frac{\sum_{d \in D} p(d) u(d)}{\left(\sum_{d \in D} p(d)\right)^{2}} \leq \frac{\sum_{b \in B} p(b) u(b)-\sum_{a \in A} p(a) u(a)}{\left(\sum_{b \in B} p(b)\right)^{2}-\left(\sum_{a \in A} p(a)\right)^{2}}=\frac{\sum_{b \in B} p(b) u(b)}{\left(\sum_{b \in B} p(b)\right)^{2}} .
$$

Hence:

$\sum_{d \in D} p(d) u(d)\left[\left(\sum_{b \in B} p(b)\right)^{2}-\left(\sum_{a \in A} p(a)\right)^{2}\right] \geq\left[\sum_{b \in B} p(b) u(b)-\sum_{a \in A} p(a) u(a)\right]\left(\sum_{d \in D} p(d)\right)^{2}$.

and

$$
\begin{gathered}
\sum_{d \in D} p(d) u(d)\left(\sum_{b \in B}(b)\right)^{2}+\sum_{a \in A} p(a) u(a)\left(\sum_{d \in D} p(d)\right)^{2} . \\
\geq \sum_{d \in D} p(d) u(d)\left(\sum_{a \in A} p(a)\right)^{2}+\sum_{b \in B} p(b) u(b)\left(\sum_{d \in D} p(d)\right)^{2}
\end{gathered}
$$

If we add (9) to this inequality, we obtain

$$
\begin{aligned}
& \sum_{a \in A} p(a) u(a)\left(\sum_{b \in B} p(b)\right)^{2}+\sum_{d \in D} p(d) u(d)\left(\sum_{b \in B} p(b)\right)^{2}+\sum_{a \in A} p(a) u(a)\left(\sum_{d \in D} p(d)\right)^{2} \\
\geq & \sum_{b \in B} p(b) u(b)\left(\sum_{a \in A} p(a)\right)^{2}+\sum_{d \in D} p(d) u(d)\left(\sum_{a \in A} p(a)\right)^{2}+\sum_{b \in B} p(b) u(b)\left(\sum_{d \in D} p(d)\right)^{2} .
\end{aligned}
$$

Let us now add $\sum_{d \in D} p(d) u(d)\left(\sum_{d \in D} p(d)\right)^{2}$ on both sides and factorize. We obtain

$$
\begin{aligned}
& {\left[\sum_{a \in A} p(a) u(a)+\sum_{d \in D} p(d) u(d)\right]\left[\left(\sum_{b \in B} p(b)\right)^{2}+\left(\sum_{d \in D} p(d)\right)\right]^{2} } \\
\geq & {\left[\sum_{b \in B} p(b) u(b)+\sum_{d \in D} p(d) u(d)\right]\left[\left(\sum_{a \in A} p(a)\right)^{2}+\left(\sum_{d \in D} p(d)\right)^{2}\right] }
\end{aligned}
$$

which implies $A \cup D \succsim B \cup D$. This concludes the proof that $\succsim$ satisfies Balancedness.

Finally, the next example shows a non-CEU ordering that satisfies richness, the Archimedean axiom and averaging but that violates Balancedness.

Example 3 Consider $\succsim$ defined on $\mathcal{P}\left(\mathbb{R}_{+}^{2}\right)$ by:

$$
A \succsim B \Longleftrightarrow \frac{\sum_{\left(a_{1}, a_{2}\right) \in A} a_{1}\left(a_{2}+\frac{a_{2}^{2}}{\sum_{\left(\alpha_{1}, \alpha_{2}\right) \in A} \alpha_{2}}\right)}{\sum_{\left(a_{1}, a_{2}\right) \in A}\left(a_{2}+\frac{a_{2}^{2}}{\sum_{\left(\alpha_{1}, \alpha_{2}\right) \in A} \alpha_{2}}\right)} \geq \frac{\sum_{\left(b_{1}, b_{2}\right) \in B} b_{1}\left(b_{2}+\frac{b_{2}^{2}}{\sum_{\left(\beta_{1}, \beta_{2}\right) \in B} \beta_{2}}\right)}{\sum_{\left(b_{1}, b_{2}\right) \in B}\left(b_{2}+\frac{b_{2}^{2}}{\sum_{\left(\beta_{1}, \beta_{2}\right) \in B} \beta_{2}}\right)}
$$


and let, for any finite $D \subset \mathbb{R}_{+}^{2}, V(D)$ be defined by:

$$
V(D)=\frac{\sum_{\left(d_{1}, d_{2}\right) \in D} d_{1}\left(d_{2}+\frac{d_{2}^{2}}{\sum_{\left(\delta_{1}, \delta_{2}\right) \in D} \delta_{2}}\right)}{\sum_{\left(d_{1}, d_{2}\right) \in D}\left(d_{2}+\frac{d_{2}^{2}}{\sum_{\left(\delta_{1}, \delta_{2}\right) \in D} \delta_{2}}\right)}
$$

It is easy to see that this ordering satisfies the Archimedean axiom. Let us show that it satisfies Richness. For this sake, consider four sets $A, B, C, D \in$ $\mathcal{P}\left(\mathbb{R}_{+}^{2}\right)$ with $A \prec B \prec C \sim D$. Define $E=\{(x, y)\}$, with $x=V(C)$. We have $\lim _{y \rightarrow 0} V(A \cup E)=V(A)$ and $\lim _{y \rightarrow \infty} V(A \cup E)=V(E)$. Since $V$ is (Hausdorff) continuous and $V(A)<V(B)<V(E)$, there exists $y \in \mathbb{R}_{+}$such that $V(A \cup E)=V(B)$. If $(x, y) \notin A \cup C \cup D$, then we have found the set $E$ as in the statement of Richness. If $(x, y) \in A \cup C \cup D$, then consider $E=$ $\{(x, y),(z, w)\}$, with $x=z=V(C)$. We have $\lim _{y, w \rightarrow 0} V(A \cup E)=V(A)$ and $\lim _{y, w \rightarrow \infty} V(A \cup E)=V(E)$. Since $V$ is Hausdorff continuous and $V(A)<$ $V(B)<V(E)$, there exist necessarily infinitely many pairs $(y, w) \in \mathbb{R}_{+}^{2}$ such that $V(A \cup E)=V(B)$. Since $A \cup C \cup D$ is finite, at least one of these pairs is such that $E \cap(A \cup C \cup D)=\emptyset$. Hence Richness holds. Let us now show that this ordering satisfies averaging. Let $A$ and $B$ be two disjoint sets such that $A \succsim B$. One has therefore:

$$
\begin{aligned}
& V(A) \geq V(B) \\
& \Longleftrightarrow \\
& \sum_{\left(b_{1}, b_{2}\right) \in B}\left(b_{2}+\frac{b_{2}^{2}}{\sum_{\left(\beta_{1}, \beta_{2}\right) \in B} \beta_{2}}\right) V(A) \\
& \sum_{\left(a_{1}, a_{2}\right) \in A}\left(a_{2}+\frac{a_{2}^{2}}{\sum_{\left(\alpha_{1}, \alpha_{2}\right) \in A} \alpha_{2}}\right)+\sum_{\left(b_{1}, b_{2}\right) \in B}\left(b_{2}+\frac{b_{2}^{2}}{\sum_{\left(\beta_{1}, \beta_{2}\right) \in B} \beta_{2}}\right) \\
& \geq \frac{\sum_{\left(b_{1}, b_{2}\right) \in B}\left(b_{2}+\frac{b_{2}^{2}}{\sum_{\left(\beta_{1}, \beta_{2}\right) \in B} \beta_{2}}\right) V(B)}{\sum_{\left(a_{1}, a_{2}\right) \in A}\left(a_{2}+\frac{a_{2}^{2}}{\sum_{\left(\alpha_{1}, \alpha_{2}\right) \in A} \alpha_{2}}\right)+\sum_{\left(b_{1}, b_{2}\right) \in B}\left(b_{2}+\frac{b_{2}^{2}}{\sum_{\left(\beta_{1}, \beta_{2}\right) \in B} \beta_{2}}\right)} \\
& =\frac{\sum_{\left(b_{1}, b_{2}\right) \in B} b_{1}\left(b_{2}+\frac{b_{2}^{2}}{\sum_{\left(\beta_{1}, \beta_{2}\right) \in B} \beta_{2}}\right)}{\sum_{\left(a_{1}, a_{2}\right) \in A}\left(a_{2}+\frac{a_{2}^{2}}{\sum_{\left(\alpha_{1}, \alpha_{2}\right) \in A} \alpha_{2}}\right)+\sum_{\left(b_{1}, b_{2}\right) \in B}\left(b_{2}+\frac{b_{2}^{2}}{\sum_{\left(\beta_{1}, \beta_{2}\right) \in B} \beta_{2}}\right)}
\end{aligned}
$$


and (trivially):

$$
\begin{gathered}
V(A) \geq V(A) \\
\Longleftrightarrow \\
\geq \frac{\sum_{\left(a_{1}, a_{2}\right) \in A}\left(a_{2}+\frac{a_{2}^{2}}{\sum_{\left(\alpha_{1}, \alpha_{2}\right) \in A} \alpha_{2}}\right) V(A)}{\sum_{\left(a_{1}, a_{2}\right) \in A}\left(a_{2}+\frac{a_{2}^{2}}{\sum_{\left(\alpha_{1}, \alpha_{2}\right) \in A} \alpha_{2}}\right)+\sum_{\left(b_{1}, b_{2}\right) \in B}\left(b_{2}+\frac{b_{2}^{2}}{\sum_{\left(\beta_{1}, \beta_{2}\right) \in B} \beta_{2}}\right)} \\
=\frac{\sum_{\left(a_{1}, a_{2}\right) \in A}\left(a_{2}+\frac{a_{2}^{2}}{\sum_{\left(a_{1}\right) \in A} \alpha_{2}}\right) V(A)}{\left.\sum_{\left(\alpha_{1}, \alpha_{2}\right) \in A} \alpha_{2}^{2}\right)+\sum_{\left(b_{1}, b_{2}\right) \in B}\left(b_{2}+\frac{b_{2}^{2}}{\sum_{\left(\beta_{1}, \beta_{2}\right) \in B} \beta_{2}}\right)} \\
\sum_{\left(a_{1}, a_{2}\right) \in A} a_{1}\left(a_{2}+\frac{a_{2}^{2}}{\sum_{\left(\alpha_{1}, \alpha_{2}\right) \in A} \alpha_{2}}\right) \\
\sum_{\left(a_{2}+a_{2}^{2}\right.}\left(b_{2}+\frac{b_{2}^{2}}{\sum_{\left(\alpha_{1}, \alpha_{2}\right) \in A} \alpha_{2}}\right)+\sum_{\left(b_{1}, b_{2}\right) \in B}
\end{gathered}
$$

Summing inequalities (12) and (13) yields:

$$
\begin{aligned}
V(A) & \geq \frac{\sum_{\left(a_{1}, a_{2}\right) \in A} a_{1}\left(a_{2}+\frac{a_{2}^{2}}{\sum_{\left(\alpha_{1}, \alpha_{2}\right) \in A} \alpha_{2}}\right)+\sum_{\left(b_{1}, b_{2}\right) \in B} b_{1}\left(b_{2}+\frac{b_{2}^{2}}{\sum_{\left(\beta_{1}, \beta_{2}\right) \in B} \beta_{2}}\right)}{\sum_{\left(a_{1}, a_{2}\right) \in A}\left(a_{2}+\frac{a_{2}^{2}}{\sum_{\left(\alpha_{1}, \alpha_{2}\right) \in A} \alpha_{2}}\right)+\sum_{\left(b_{1}, b_{2}\right) \in B}\left(b_{2}+\frac{b_{2}^{2}}{\sum_{\left(\beta_{1}, \beta_{2}\right) \in B} \beta_{2}}\right)} \\
& =V(A \cup B)
\end{aligned}
$$

as required by the first part of Averaging. The other part of the axiom can be obtained by an analogous reasoning. Let us now show that the ordering $\succsim$ violates balancedness. Indeed, consider the sets $A=\{(505,16)\}, B=\{(10,10),(1000,10)\}$, $C=\{(504,1)\}$ and $D=\{(1,10)\}$ one has:

$$
\begin{aligned}
& V(A)=\frac{\sum_{\left(a_{1}, a_{2}\right) \in A} a_{1}\left(a_{2}+\frac{a_{2}^{2}}{\sum_{\left(\alpha_{1}, \alpha_{2}\right) \in A} \alpha_{2}}\right)}{\sum_{\left(a_{1}, a_{2}\right) \in A}\left(a_{2}+\frac{a_{2}^{2}}{\sum_{\left(\alpha_{1}, \alpha_{2}\right) \in A} \alpha_{2}}\right)}=505=\frac{10 \times\left(10+\frac{100}{10+10}\right)+1000 \times\left(10+\frac{100}{10+10}\right)}{\left(10+\frac{100}{10+10}\right)+\left(10+\frac{100}{10+10}\right)}= \\
& V(B)>V(C)=\frac{504 \times\left(1+\frac{1}{1}\right)}{1+\frac{1}{1}}=504>V(D)=\frac{1 \times\left(10+\frac{100}{10}\right)}{10+\frac{100}{10}}=1
\end{aligned}
$$

One has also: 


$$
\begin{aligned}
V(A \cup C)= & \frac{505 \times\left(16+\frac{256}{17}\right)+504 \times\left(1+\frac{1}{17}\right)}{\left(16+\frac{256}{17}\right)+\left(1+\frac{1}{17}\right)}=\frac{45952}{91} \simeq 504.97 \\
V(B \cup C) & =\frac{10 \times\left(10+\frac{100}{10+10+1}\right)+1000 \times\left(10+\frac{100}{10+10+1}\right)+504 \times\left(1+\frac{1}{10+10+1}\right)}{\left(10+\frac{100}{10+10+1}\right)+\left(10+\frac{100}{10+10+1}\right)+\left(1+\frac{1}{10+10+1}\right)} \\
& =\frac{10 \times\left(10+\frac{100}{21}\right)+1000 \times\left(10+\frac{100}{21}\right)+504 \times\left(1+\frac{1}{21}\right)}{21+\frac{201}{21}} \\
& \simeq 504.97 .
\end{aligned}
$$

However, contrary to what balancedness requires:

$$
\begin{aligned}
V(A \cup D) & =\frac{505 \times\left(16+\frac{256}{26}\right)+1 \times\left(1+\frac{1}{26}\right)}{\left(16+\frac{256}{26}\right)+\left(1+\frac{1}{26}\right)} \\
& \simeq 485.53 \\
& <V(B \cup D) \\
& =\frac{10 \times\left(10+\frac{100}{10+10+10}\right)+1000 \times\left(10+\frac{100}{10+10+10}\right)+1 \times\left(1+\frac{1}{10+10+10}\right)}{\left(10+\frac{100}{10+10+10}\right)+\left(10+\frac{100}{10+10+10}\right)+\left(1+\frac{1}{10+10+10}\right)} \\
& \simeq 486.20
\end{aligned}
$$

\subsection{Some unpleasant implications of our richness condi- tion}

The richness condition used in our characterization is strong. Among other things, it seems to restrict unduly the functions $p$ and $u$ that appear in the representation of a CEU criterion. For the moment, we can not analytically identify what these additional restrictions - beyond that of being functions from $X$ to the real (and for the $p$ function, to have strictly positive range). We can not either provide a topological interpretation of our characterization result in a similar spirit than the one obtained in Gravel, Marchant, and Sen (2012). An example of the implication of our richness condition is provided in the following proposition, where we show that if $X=\mathbb{R}$ (for instance the consequences of a decision under ignorance are amounts of money), then it is impossible with our richness condition to have both the functions $p$ and the function $u$ to be monotonic if the function $u$ is continuous.

Proposition 1 Suppose that $X=\mathbb{R}$. Then if $\succsim$ is a CEU ranking satisfying richness, then, if the function $u$ in expression (1) is continuous, it can not be monotonic if $p$ is monotonic.

In the next proposition, we establish that if $X$ is a topological space (for instance a separable one of the kind considered in Gravel, Marchant, and Sen (2012)), then no Uniform Expected Utility criterion in which $u$ is a continuous utility function satisfies the richness condition. This shows that the characterization of the CEU family of criteria that we provide in this paper does not 
contain all members of that family because it excludes, at least in topological environments, the UEU subclass of that family that is obtained by considering only constant functions $p$ and continuous functions $u$.

Proposition 2 Let $X$ be a topological space, and let $\succsim$ is a non-trivial UEU ranking with $u$ continuous. Then $\succsim$ violates the Richness condition.

\section{Conclusion}

This paper characterizes the family of CEU rankings of decisions under ignorance or objective ambiguity with finitely many consequences, under the assumption that the rankings are defined in a "rich" environment. With the exception perhaps of the Archimedean axiom, the two main axioms used in the characterization, averaging and balancedness, that also appear in the characterization of a similar family obtained by Ahn (2008), are easy to interpret and to test in an experimental context. As we argued above, the fact that we limit our attention to decision with finitely many consequences (or probability distributions) makes our framework much more applicable that the atomless environment considered by Ahn (2008) and the literature that derives from the Bolker-Jeffrey tradition (e.g. Bolker (1966), Bolker (1967), Jeffrey (1965) and Broome (1990)). We emphasize also that the discrete framework makes the proof and the characterization very different from the one obtained in this later tradition. Moreover, we have shown that the three axioms that we use are independent.

Yet, the analysis conducted in this paper suffers from two limitations, of varying importance. First, as suggested in the preceding section, it rides on a richness assumption that is, probably, unduly strong. We use the qualifier "probably" because we do not have, at the moment, an alternative. We are therefore incapable to assess the strength of the assumption. But, as shown in Proposition 2, the richness condition is sufficiently strong for excluding from the family of CEU rankings all UEU ones of the kind characterized in Gravel, Marchant, and Sen (2012) when the later are defined on a topological space and are continuous on that space. Another limitation of the analysis is that it is conducted in an algebraic framework rather than a topological one (using Wakker (1988)'s terminology). Contrary to what was achieved in Gravel, Marchant, and Sen (2012), we did not succeed indeed in providing a topological version of our theorem in which richness and the Archimedean condition could be replaced by an appropriate - and necessary - continuity condition. We do not view this second limitation as being as important as the first however. Indeed, as very convincingly argued - at least in our view - by Wakker (1988), the algebraic framework is more general than the topological one. Yet, it is fair to say that topological environments, and the continuity properties that they enable to define, are more familiar to decision theorists and economists than richness and Archimedean conditions. For this reason, it would be nice to have a topological version of our main theorem. 
The analysis of this paper needs also to be completed in several directions. One of them is an analysis of comparative attitudes toward radical uncertainty or toward ambiguity in the class of CEU rankings. Another one is a proper understanding of the uniqueness properties of the functions $u$ and $\rho$ used in the characterization. As shown in Proposition 1, the two functions may not be totally independent from each others in specific environment. It would be nice to have a complete identification of the uniqueness properties of these functions. Finally, we believe that CEU models of decision making under radical uncertainty and/or ambiguity should be put to work, notably in public policies, to generate consistent rankings of radically uncertain decisions. While environmental policy discussed in Introduction is an obvious fields for such applications, there are many others. We plan to develop such applications in our future work.

\section{Appendix: Proofs}

\subsection{Lemma 1}

Let the sets $A$ and $C \in \mathcal{P}(X)$ be such that $A \succsim B \succsim C$ for all sets $B \in \mathcal{P}(X)$ and assume by contradiction that there exists some consequence $x \in A \cap C$. Since $\succsim$ is not trivial, one must have $A \succ C$. Since $\succsim$ is an ordering, either $\{x\} \succsim A \succ C$ or $A \succ\{x\}$. In the first case, it follows from Averaging that $C \succ C \backslash\{x\}$, which contradicts the definition of the sets $A$ and $C \in \mathcal{P}(X)$ to be such that $A \succsim B \succsim C$ for all sets $B \in \mathcal{P}(X)$. In the second case, it follows from Averaging again that $A \backslash\{x\} \succ A$ which is also a contradiction of the definition of the sets $A$ and $C \in \mathcal{P}(X)$ to be such that $A \succsim B \succsim C$ for all sets $B \in \mathcal{P}(X)$.

\subsection{Lemma 2.}

Since $\succsim$ is non-trivial, there is a set $D$ such that $D \prec A$ or $A \prec D$. We treat the case $A \prec D$ (the other case is handled symmetrically). We first prove that there are at least two equivalence classes better than the one containing $A$, so that it will be possible to apply Richness. We consider two cases :

(1) $A \cap D=\varnothing$. Then Averaging yields $A \prec A \cap D \prec D$ (and we are done).

(2) $A \cap D \neq \varnothing$. We then consider three subcases:

(a) $A \cap D \sim A$. Then, by Averaging, $A \backslash D \sim A$ and $A \backslash D \prec A \cup D \prec D$.

(b) $A \cap D \prec A$. If this is the case, one can not have $D \subset A$. Indeed, if one had $D \subset A$, this would imply that $A \cap D=D \prec A$, which contradicts the initial assumption that $A \prec D$. Hence the set $D \backslash A \neq \varnothing$. Averaging then implies that $D \prec D \backslash A$.

(c) $A \cap D \succ A$. If $A \cap D \nsucc D$, then we are done. Otherwise, by Averaging, $D \backslash A \sim D$ and $A \prec A \cup(D \backslash A) \prec D \backslash A$.

We now apply richness to the three equivalence classes. A first application of Richness yields a set $B_{1}$ such that $B_{1} \sim A$ and $B_{1} \cap A=\varnothing$. If $B_{1} \cap C=\varnothing$, then the proof is done. If $B_{1} \cap C \neq \varnothing$, then use Richness again to find a set $B_{2}$ such that $B_{2} \sim A \cup B_{1}$ and $B_{2} \cap\left(A \cup B_{1}\right)=\varnothing$. By Averaging, $A \cup B_{1} \sim A$ and, by transitivity, $B_{2} \sim A$. We are now sure that $B_{2}$ does not contain any of the elements of $B_{1} \cap C$. If 
$B_{2} \cap C=\varnothing$, then the proof is done. If $B_{2} \cap C \neq \varnothing$, then use Richness again to find a set $B_{3}$ such that $B_{3} \sim A \cup B_{1} \cup B_{2}$ and $B_{3} \cap\left(A \cup B_{1} \cup B_{2}\right)=\varnothing$. By Averaging, $A \cup B_{1} \cup B_{2} \sim A$ and, by transitivity, $B_{3} \sim A$. Notice that $\left(B_{1} \cup B_{2}\right) \cap C \supsetneq B_{1} \cap C$ . We are now sure that $B_{3}$ does not contain any of the elements of $\left(B_{1} \cup B_{2}\right) \cap C$. If $B_{3} \cap C=\varnothing$, then the proof is done. If $B_{3} \cap C \neq \varnothing$, we iterate this construction and we find sets like $B_{4}, B_{5}, \ldots$ At each iteration, $\left(B_{1} \cup \ldots \cup B_{i}\right) \cap C \supsetneq\left(B_{1} \cup \ldots \cup B_{i-1}\right) \cap C$ . Since $C$ is finite, we are sure to reach some $B_{j}$ satisfying the same conditions as $B$ in the statement of the lemma.

\subsection{Lemma 3}

If $\succsim$ is not trivial, then there are decisions $D$ and $E \in \mathcal{P}(X)$ such that $D \prec E$. By Lemma 2, there is a set $F \in \mathcal{P}(X)$ such that $F \sim D$ and $F \cap(D \cup E)=\varnothing$. By Averaging and Transitivity, $D \prec F \cup E \prec E$. Hence, the ordering $\succsim$ has at least three equivalence classes and, hence, $\mathcal{P}_{*}(X)$ is not empty. Let $B$ be a decision in $\mathcal{P}_{*}(X)$ (we have just proved that it exists). We will prove that there is $A \in \mathcal{P}_{*}(X)$ such that $A \prec B$ (the proof that there is $C \in \mathcal{P}_{*}(X)$ such that $B \prec C$ is similar). If $m(X)$ is empty, then the proof is immediate. So, we consider that $m(X)$ is not empty. Let $G$ be a decision in $m(X)$. By Lemma 2, there is a set $H \in P(X)$ such that $H \sim G$ and $H \cap(G \cup B)=\varnothing$. By Averaging and Transitivity, $H \prec H \cup B \prec B$.

\subsection{Lemma 4}

Let $A, B$, and $C$ be three sets in $\mathcal{P}^{E}(X)$ such that $A \succsim_{l} B$ and $B \succsim_{l} C$. By definition of $\succsim_{l}$, this implies the existence of sets $D$ and $D^{\prime} \in \mathcal{P}_{*}(X)$ respectively disjoint from $A \cup B$ and $B \cup C$ such that $E \succ D, D^{\prime}, A \cup D \succsim B \cup D$ and $B \cup D^{\prime} \succsim C \cup D^{\prime}$. Thanks to Lemma 2, we can find a set $D^{\prime \prime} \in \mathcal{P}_{*}(X)$ disjoint from $A \cup B \cup C$, with $D \sim D^{\prime \prime}$. By Balancedness, $A \cup D^{\prime \prime} \succsim B \cup D^{\prime \prime}$ and $B \cup D^{\prime \prime} \succsim C \cup D^{\prime \prime}$. By transitivity, $A \cup D^{\prime \prime} \succsim C \cup D^{\prime \prime}$ and, hence, $A \succsim_{l} C$. This proves the transitivity of $\succsim_{l}$. As for completeness, let $A$ and $B$ be two sets in $P^{E}(X)$ such that $A \succsim_{l} B$. By definition of $\succsim_{l}$, either:

(i) there is no set $C$ disjoint from $A \cup B$ such that $E \succ C$ or :

(ii) there are such sets but for none of them it is true that $A \cup C \succsim B \cup C$.

Case (i) can be ruled out by Lemma 3 . If case (ii) holds, then, since $\succsim$ is complete, we must have $A \cup C \prec B \cup C$ for all sets $C$ disjoint from $A \cup B$ such that $E \succ C$. It follows that $B \succsim_{l} A$ and the relation $\succsim_{l}$ is therefore complete.

\subsection{Lemma 5}

For the "only if" part of the first part of the lemma, we know that, since $\succsim^{l}$ is complete, $A \succ_{l} B$ implies $B \succsim_{l} A$. Hence, either there is no $D$ disjoint from $A \cup B$ with $D \prec A$ or $B \cup D \prec A \cup D$ for all sets $D \prec A$. The first of these two possibilities is ruled out by Lemma 3. The second one implies, as a particular case, that $A \cup C \succ B \cup C$. For 
the "if" part of the first part of the lemma, suppose $A \cup C \succ B \cup C$. This implies $A \succsim_{l} B$ (by definition of $\succsim_{l}$ ). Suppose by contradiction that $A \succ_{l} B$ does not hold. Since $\succsim_{l}$ is complete, $B \succsim_{l} A$ must hold so that, by definition of $\succsim_{l}$, there exists a set $D$ such that $B \cup D \succsim A \cup D$, and $D \prec A$. But this contradicts balancedness. Hence $A \succ_{l} B$ must hold.

For the "only if part of the second part of the lemma, one knows that $A \sim_{l} B$ implies the existence of sets $D$ and $D^{\prime}$ (both strictly dominated by $A$ as per $\succsim$ ) such that $\left(D \cup D^{\prime}\right) \cap(A \cup B)=\varnothing, A \cup D \succsim B \cup D$ and $B \cup D^{\prime} \succsim A \cup D^{\prime}$. By balancedness,

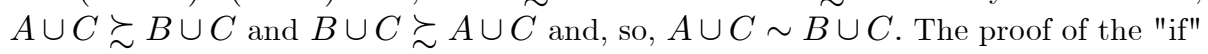
part of the second part of the lemma is obvious.

\subsection{Lemma 6}

Define the binary operation ${ }^{E}$ on $\mathcal{P}^{E}(X)$ as follows. If $A \cap B=\varnothing$, then $A \circ^{E} B=$ $A \cup B$. Otherwise set $A \circ^{E} B=A^{\prime} \cup B^{\prime}$ for some $A^{\prime}, B^{\prime} \in \mathcal{P}^{E}(X)$ such that $A^{\prime} \cap B^{\prime}=\varnothing, A^{\prime} \cup C \sim A \cup C$ and $B^{\prime} \cup D \sim B \cup D$ for some $C, D \prec E$ such that $\left(A \cup A^{\prime}\right) \cap C=\varnothing$ and $\left(B \cup B^{\prime}\right) \cap D=\varnothing$. The existence of such sets $A^{\prime}, B^{\prime}$ does not pose any difficulty, thanks to Richness. Indeed, by Lemma 3 and Averaging, there exists a set $C \in \mathcal{P}(X)$ such that $C \prec A \sim B$. By Averaging, $C \prec C \cup A \prec A$ and, using Richness, there exists a set $A^{\prime}$ such that $A^{\prime} \cup C \sim A \cup C, A^{\prime} \sim A$ and $A^{\prime} \cap(C \cup A)=\varnothing$. Using an analogous reasoning, one can establish the existence of a set $B^{\prime}$ such that $B^{\prime} \cup C \sim B \cup C, B^{\prime} \sim B$ and $B^{\prime} \cap\left(C \cup A \cup A^{\prime}\right)=\varnothing$.

Hence $\circ^{E}$ is defined for every pair $A, B \in \mathcal{P}^{E}(X)$, and the choice of the sets $A^{\prime}$ and $B^{\prime}$ can be made by any rule whatsoever if there are several such sets for a given pair $A$ and $B$. Finally we note that ${ }^{E}$ is closed in the set $\mathcal{P}^{E}(X)$ thanks to Averaging.

For any $E \in \mathcal{P}(X)$, we now show that the structure formed by the set $\mathcal{P}^{E}(X)$, the binary relation $\succsim_{l}$ and the binary operation $\circ^{E}$ is what Krantz, Luce, Suppes, and Tversky (1971) (p. 73, definition 1) call a closed extensive measurement structure. That is to say, we establish that:

1. $\succsim_{l}$ is a weak order: see Lemma 4 ;

2. $\circ^{E}$ is weakly associative so that $A \circ^{E}\left(B \circ^{E} C\right) \sim_{l}\left(A \circ^{E} B\right) \circ^{E} C$ for every $A, B$ and $C \in \mathcal{P}^{E}(X)$. The proof of this is obvious if $A, B, C$ are mutually disjoint. Consider now the case where $A \cap B \cap C \neq \varnothing$. Let $A^{\prime}, B^{\prime}, C^{\prime} \in \mathcal{P}(X)$ be mutually disjoint sets such that $A^{\prime} \cup M \sim A \cup M, B^{\prime} \cup N \sim B \cup N$, $C^{\prime} \cup O \sim C \cup O$ for some $M, N, O \prec E$ with $\left(A \cup A^{\prime}\right) \cap M=\left(B \cup B^{\prime}\right) \cap N=$ $\left(C \cup C^{\prime}\right) \cap O=\varnothing$. They exist thanks to Richness (the argument is similar to that employed in the definition of the binary operation $\left.{ }^{E}{ }^{E}\right)$. We have $B{ }^{E}{ }^{E} C=$ $B^{\prime} \cup C^{\prime}$ and $A \circ^{E}\left(B \circ^{E} C\right)=A^{\prime} \cup B^{\prime} \cup C^{\prime}$. We also have $A \circ^{E} B=A^{\prime} \cup B^{\prime}$ and $\left(A \circ^{E} B\right) \circ^{E} C=A^{\prime} \cup B^{\prime} \cup C^{\prime}$, so that $A \circ^{E}\left(B \circ^{E} C\right)=\left(A \circ^{E} B\right) \circ^{E} C$. The reasoning is similar when some but not all pairwise intersections between $A, B, C$ are not empty. 
3. monotonicity holds (that is: $A \succsim_{l} B$ iff $A \circ^{E} C \succsim_{l} B \circ^{E} C$ iff $C \circ^{E} A \succsim_{l}$ $C \circ^{E} B$ ). Since $\circ^{E}$ is obviously commutative, we just need to prove $A \succsim_{l} B$ iff $A \circ^{E} C \succsim_{l} B \circ^{E} C$. Choose $A^{\prime}$ and $B^{\prime}$ in $\mathcal{P}^{E}(X)$ such that $A^{\prime} \cap C=\varnothing=B^{\prime} \cap C$, $A^{\prime} \cup D \sim A \cup D$ and $B^{\prime} \cup D \sim B \cup D$ for some $D \prec E$ and disjoint from $C$. Thanks to Richness, this is always possible. Notice that $C \cup D \prec E$ by averaging. We have $A \succsim_{l} B$ iff $A \cup F \succsim B \cup F$ (by definition) iff $A^{\prime} \cup D \succsim B^{\prime} \cup D$ (by construction) iff $A^{\prime} \cup C \cup D \succsim B^{\prime} \cup C \cup D$ (by Balancedness and because $C \cup D \prec E$ thanks to Averaging) iff $A \circ^{E} C \succsim_{l} B \circ^{E} C$;

4. The Archimedean axiom: if $A \succ_{l} B$, then, for any $C, D \in \mathcal{P}^{E}(X)$, there exists a positive integer $n$ such that $n . A \circ^{E} C \succsim_{l} n . B \circ^{E} D$, where $n . A$ is defined inductively as: $1 . A=A,(n+1) \cdot A=n \cdot A \circ^{E} A$. It is immediate to see that this condition is implied by the Archimedean axiom.

By Theorem 1 of Krantz, Luce, Suppes, and Tversky (1971) (p.74), for any $E \in$ $\mathcal{P}_{*}(X)$, there exists a mapping $p^{E}: \mathcal{P}^{E}(X) \rightarrow \mathbb{R}$ such that, for all $A, B \in \mathcal{P}^{E}(X)$, $A \succsim_{l} B$ iff $p^{E}(A) \geq p^{E}(B)$ and $p^{E}\left(A \circ^{E} B\right)=p^{E}(A)+p^{E}(B)$. Furthermore, $p^{E}$ is unique up to a linear transformation.

We now show that $p^{E}(A)>0$ for all $A \in \mathcal{P}^{E}(X)$. For any $A \in \mathcal{P}^{E}(X)$, we can find a set $B \in \mathcal{P}^{E}(X)$ such that $A \cap B=\varnothing$ (using Lemma 2). By definition of $\mathcal{P}_{*}(X)$, there is $D^{\prime} \prec E$. By Lemma 2, there is $D \sim D^{\prime} \prec E$ such that $D \cap(A \cup B)=\varnothing$. By Averaging, $D \prec B \cup D \prec B \sim A$. By Averaging again, $B \cup D \prec B \cup D \cup A \prec B$. By definition of $\succsim_{l}, B \prec_{l} A \cup B$. This implies $p^{E}(B)<p^{E}(A \cup B)$ and, since $A$ and $B$ are disjoint, $p^{E}(B)<p^{E}(A)+p^{E}(B)$ or, equivalently, $p^{E}(A)>0$.

\section{7 $\quad$ Lemma 7}

For a fixed $E \in \mathcal{P}_{*}(X)$, let $\mathcal{L}=\{a \in X:\{a\} \prec E\}$ and $\mathcal{U}=\{a \in X:\{a\} \succ E\}$. These sets are not empty (this is an almost immediate consequence of Lemma 3 ). Define $M$ as an arbitrary set such that $E \prec M$.

We first define $\nu^{E}$ on $\mathcal{P}(\mathcal{L})$. Fix some $L \in \mathcal{L}$. By Richness, there is $U \in \mathcal{P}(\mathcal{U})$ such that $U \sim M$ and $U \cup L \sim E$. Set $\nu^{E}(L)=-p^{M}(U)$. By construction, $\nu^{E}(L)$ does not depend on the choice of $U$. Indeed, suppose there are several such $U$, say $U$ and $U^{\prime}$. Notice that $U \sim M \sim U^{\prime}, U \cup L \sim E$ and $U^{\prime} \cup L \sim E$. So, $U \cup L \sim U^{\prime} \cup L$. Hence $U \sim_{l} U^{\prime}$ and $p^{M}(U)=p^{M}\left(U^{\prime}\right)$.

Select $L_{1}, L_{2} \in \mathcal{L}$, with $L_{1} \cap L_{2}=\varnothing$. By Averaging, $L_{1} \cup L_{2} \in \mathcal{L}$. Using Richness as above, we find two disjoint sets $U_{1}, U_{2} \in \mathcal{P}(\mathcal{U})$ such that $U_{1} \sim U_{2} \sim M$, $U_{1} \cup L_{1} \sim E$ and $U_{2} \cup L_{2} \sim E$. By Averaging, $U_{1} \cup U_{2} \cup L_{1} \cup L_{2} \sim E, U_{1} \cup U_{2} \sim M$ and $U_{2} \cup L_{2} \sim E$. So,

$$
\begin{aligned}
\nu^{E}\left(L_{1} \cup L_{2}\right) & =-p^{M}\left(U_{1} \cup U_{2}\right) \\
& =-p^{M}\left(U_{1}\right)-p^{M}\left(U_{2}\right) \\
& =\nu^{E}\left(L_{1}\right)+\nu^{E}\left(L_{2}\right) .
\end{aligned}
$$

This proves that $\nu^{E}$ is disjoint-additive over $\mathcal{L}$.

We now define $\nu^{E}$ on $\mathcal{P}(\mathcal{U})$. Take any $U \in \mathcal{P}(\mathcal{U})$. By Richness used in a similar (but this time "downward") way as above, there is $L \in \mathcal{P}(\mathcal{L})$ such that $U \cup L \sim E$. 
Set $\nu^{E}(U)=-\nu^{E}(L)$. The mapping $\nu^{E}$ on $\mathcal{P}(\mathcal{U})$. does not depend on the choice of $L$. Indeed, suppose there are several such $L$, say $L_{1}$ and $L_{2} \operatorname{in} \mathcal{P}(\mathcal{L})$. We must prove that $\nu^{E}\left(L_{1}\right)=\nu^{E}\left(L_{2}\right)$. Suppose first $L_{1} \cap L_{2}=\emptyset$. Let $U_{1}, U_{2} \in \mathcal{P}(\mathcal{U})$ be such that $U_{1} \cap U=\emptyset=U_{2} \cap U, U_{1} \sim M \sim U_{2}, U_{1} \cup L_{1} \sim E \sim U_{2} \cup L_{2}$. By Richness, such sets exist. We also have $U \cup L_{1} \sim E \sim U \cup L_{2}$. By Averaging, $U_{1} \cup L_{1} \cup U \cup L_{2} \sim E \sim U_{2} \cup L_{2} \cup U \cup L_{1}$. Hence, $U_{1} \sim_{l} U_{2}, p^{M}\left(U_{1}\right)=p^{M}\left(U_{2}\right)$ and $\nu^{E}\left(L_{1}\right)=\nu^{E}\left(L_{2}\right)$. Suppose now $L_{1} \cap L_{2} \neq \emptyset$. By Richness used in the same way as above, there is $L_{3} \in \mathcal{P}(\mathcal{L})$ such that $L_{3} \cap\left(L_{1} \cup L_{2}\right)=\emptyset$ and $U \cup L_{3} \sim E$. Define $U_{3}$ by $U_{3} \sim M$ and $U_{3} \cup L_{3} \sim E$. By richness, $U_{3}$ can be chosen disjoint from both $U_{1}$ and $U_{2}$. Since $U_{1} \cup L_{1} \sim U \cup L_{3} \sim E \sim U_{3} \cup L_{3} \sim U \cup L_{1}$ and $U, U_{1}$ and $U_{3}$ are disjoint as are $L_{1}$ and $L_{2}$, it follows from Averaging that $U_{1} \cup L_{1} \cup U \cup L_{3} \sim E \sim U_{3} \cup L_{3} \cup U \cup L_{1}$. Hence, $U_{1} \sim_{l} U_{3}$ and, therefore, $p^{M}\left(U_{1}\right)=p^{M}\left(U_{3}\right)$. A similar reasoning can be performed for $U_{2}$ and $U_{3}$. We therefore have $p^{M}\left(U_{1}\right)=p^{M}\left(U_{2}\right)=p^{M}\left(U_{3}\right)$ and, as a result, $\nu^{E}\left(L_{1}\right)=\nu^{E}\left(L_{3}\right)=\nu^{E}\left(L_{2}\right)$.

The mapping $\nu^{E}$ on $\mathcal{P}(\mathcal{U})$ is additive. Indeed, consider two sets $U_{1}, U_{2} \in \mathcal{P}(\mathcal{U})$, with $U_{1} \cap U_{2}=\emptyset$. Let us find two sets $L_{1}, L_{2} \in \mathcal{P}(\mathcal{L})$ such that $U_{1} \cup L_{1} \sim E \sim$ $U_{2} \cup L_{2}$. Since the choice of $L_{1}$ and $L_{2}$ is not important, we can choose them disjoint (using Richness). By Averaging, $U_{1} \cup U_{2} \cup L_{1} \cup L_{2} \sim E$. So, $\nu^{E}\left(U_{1} \cup U_{2}\right)=$ $-\nu^{E}\left(L_{1} \cup L_{2}\right)=-\nu^{E}\left(L_{1}\right)-\nu^{E}\left(L_{2}\right)=\nu^{E}\left(U_{1}\right)+\nu^{E}\left(U_{2}\right)$.

We define then $\nu^{E}$ on the whole set $P(X)$. Take any $S \in P(X)$. If $\{s\} \sim E$ for all $s \in S$, set $\nu^{E}(S)=0$. Otherwise, we can express $S$ as $S=L \cup U \cup R$ with $L=S \cap \mathcal{L}, U=S \cap \mathcal{U}$ and $R=S \backslash(\mathcal{L} \cup \mathcal{U})$. By Averaging, $S \succsim E$ iff $L \cup U \succsim E$. Set $\nu^{E}(S)=\nu^{E}(L)+\nu^{E}(U)$. Disjoint-additivity is inherited from $\nu^{E}$ on $\mathcal{P}(\mathcal{U})$ and $\nu^{E}$ on $\mathcal{P}(\mathcal{L})$.

We must finally check whether $\nu^{E}$ satisfies (ii). Suppose $S \succ E$. Then $(S \cap \mathcal{L}) \cup$ $(S \cap \mathcal{U}) \succ E$. Using richness and averaging, one can find a superset $L^{\prime}$ of $S \cap \mathcal{L}$ belonging to $\mathcal{P}(\mathcal{L})$ such that $L^{\prime} \cup(S \cap \mathcal{U}) \sim E$. As shown above, $-v^{E}\left(L^{\prime E}(S \cap \mathcal{U})\right.$. Since $S \cap \mathcal{L} \subset L^{\prime} \subseteq \mathcal{L}$, and, for every $L \in \mathcal{P}(\mathcal{L}), \nu^{E}(L)=-p^{M}(U)<0$ for some set $U \in \mathcal{P}(\mathcal{U})$ we have that $0>\nu^{E}(S \cap \mathcal{L})>\nu^{E}\left(L^{\prime}\right)$ by disjoint-additivity. Now, by construction, $\nu^{E}(S)=\nu^{E}(S \cap \mathcal{L})+\nu^{E}(S \cap \mathcal{U})=\nu^{E}(S \cap \mathcal{L})-\nu^{E}\left(L^{\prime}\right)>0$.

Suppose now $S \prec E$. Then $(S \cap \mathcal{L}) \cup(S \cap \mathcal{U}) \prec E$. Using Averaging and Richness again, there is a superset $U^{\prime}$ of $S \cap \mathcal{U}$ belonging to $\mathcal{P}(\mathcal{U})$ such that $U^{\prime} \cup$ $(S \cap \mathcal{L}) \sim E$. By definition of the mapping $\nu^{E}$, one has that $\nu^{E}\left(U^{\prime E}(S \cap \mathcal{L})>0\right.$. Moreover, since $S \cap \mathcal{U} \subset U^{\prime} \subseteq \mathcal{U}$ and $\nu^{E}(U)>0$ for every $U \in \mathcal{P}(\mathcal{U})$, one has $\nu^{E}\left(U^{\prime}\right)>V^{E}(S \cap \mathcal{U})>0$ by disjoint-additivity. We have, by construction, $\nu^{E}(S)=$ $\nu^{E}(S \cap \mathcal{L})+\nu^{E}(S \cap \mathcal{U})=\nu^{E}(S \cap \mathcal{U})-\nu^{E}\left(U^{\prime}\right)<0$

Suppose finally $S \sim E$. Then $(S \cap \mathcal{L}) \cup(S \cap \mathcal{U}) \sim E$ so that $\nu^{E}(S \cap \mathcal{L})=$ $-\nu^{E}(S \cap \mathcal{U})$. We have, by construction, $\nu^{E}(S)=\nu^{E}(S \cap \mathcal{L})+\nu^{E}(S \cap \mathcal{U})=$ $\nu^{E}(S \cap \mathcal{U})-\nu^{E}(S \cap \mathcal{U})=0$.

\subsection{Lemma 8}

Take any $A, B \in \mathcal{P}_{*}(X)$. The result is immediate if $A \sim B$. We provide the proof for the case where $A \succ B$ (the argument for the case where $B \succ A$ being symmetric. We must establish that, for any two sets $S$ and $T \in \mathcal{P}^{A}(X)$ one has $S \succsim^{l} T \Longleftrightarrow \nu^{B}(S) \geq \nu^{B}(T)$. By definition of the ordering $\succsim^{l}$ this amounts to 
showing that establish that

$$
\nu^{B}(S) \geq \nu^{B}(T) \Longleftrightarrow S \cup F \succsim T \cup F .
$$

holds for every $S$ and $T$ such that $S \sim T \sim A$ and every $F \prec A$. Consider indeed such sets $S$ and $T$ with $A \sim S \sim T$. By Lemma 7, $\nu^{A}(S)=0=\nu^{A}(T)$. By construction, $\nu^{B}(S)>0$. By richness and the fact that $A$ and $B \in \mathcal{P}_{*}(X)$, one can find a set $L_{1}$ such that $L_{1} \cap(S \cup T)=\varnothing$ and $S \cup L_{1} \sim B$. By Averaging $L_{1} \prec B$. By Lemma $7, \nu^{B}(S)+\nu^{B}\left(L_{1}\right)=\nu^{B}\left(S \cup L_{1}\right)=0$. Suppose $\nu^{B}(T) \geq \nu^{B}(S)$. Then, $\nu^{B}\left(T \cup L_{1}\right)=\nu^{B}(T)+\nu^{B}\left(L_{1}\right) \geq 0$. By Lemma 7, $T \cup L_{1} \succsim B \sim S \cup L_{1}$. By Balancedness, $T \cup F \succsim S \cup F$ for any $F: F \prec A, F \cap(S \cup T)=\varnothing$. A similar argument shows that $\nu^{B}(T)>\nu^{B}(S) \Rightarrow T \cup F \succ S \cup F$ for any $F: F \prec A, F \cap(S \cup T)=\varnothing$.

Conversely, suppose $S \cup F \succsim T \cup F$ for some $F: F \prec A, F \cap(S \cup T)=\varnothing$. By Richness, there is $L_{2}$ such that $L_{2} \cap(S \cup T)=\varnothing, T \cup L_{2} \sim B$. By Averaging, $L_{2} \prec B$. By Balancedness, $S \cup L_{2} \succsim T \cup L_{2} \sim B$. By Lemma $7, \nu^{B}(S)+\nu^{B}\left(L_{2}\right) \geq 0$. Since $\nu^{B}(T)+\nu^{B}\left(L_{2}\right)=0$, we obtain $\nu^{B}(S) \geq \nu^{B}(T)$. The same argument holds if we suppose $T \cup F \succ S \cup F$, and this establishes (14) and, therefore, the proof of the lemma.

\section{$6.9 \quad$ Lemma 9}

Consider any two sets $A$ and $B$ such that $A \succ B$ and choose some sets $S, T \in P^{A}(X)$ and $B, D \prec A$ in such a way that $D \cap(S \cup T)=\varnothing$. By Richness, this choice is possible. Suppose without loss of generality that $S \cup D \precsim T \cup D$. By iterative application of Richness, there exist sets $S_{1}, S_{2}, \ldots$ such that, for every $i \neq j \in N$, $S \cap\left(\bigcup_{i \in \mathbb{N}} S_{i}\right)=\varnothing=S_{i} \cap S_{j}=S_{i} \cap D, S_{i} \sim S$ and $\nu^{B}(S)=\nu^{B}\left(S_{i}\right)$. Similarly, there exist $T_{1}, T_{2}, \ldots$ such that, for every $i \neq j \in N, T \cap\left(\bigcup_{i \in \mathbb{N}} T_{i}\right)=\varnothing=T_{i} \cap T_{j}=T_{i} \cap D$, $T_{i} \sim T$ and $\nu^{B}(T)=\nu^{B}\left(T_{i}\right)$.

For every positive integer $n$, there is a largest integer $q(n)$ such that $\bigcup_{i=1}^{q(n)} S_{i} \cup$ $D \precsim \bigcup_{i=1}^{n} T_{i} \cup D$ because $\nu^{B}\left(\bigcup_{i=1}^{p} S_{i}\right)=p \nu^{B}\left(S_{i}\right)$ (remember that $\nu^{B}$ is additive) and is therefore unbounded when $p$ increases. Notice that $q(n) \geq n$ because $\nu^{B}(S) \leq \nu^{B}(T)$. We thus have $\bigcup_{i=1}^{q(n)} S_{i} \cup D \precsim \bigcup_{i=1}^{n} T_{i} \cup D \prec \bigcup_{i=1}^{q(\bar{n})+1} S_{i} \cup D$, for every positive integer $n$. Since the sets $\bigcup_{i=1}^{q(n)} S_{i}, \bigcup_{i=1}^{n} T_{i}$ and $\bigcup_{i=1}^{q(n)+1} S_{i}$ are all equivalent to $A$ (by Averaging) and thanks to Lemma 8, we have $\nu^{B}\left(\bigcup_{i=1}^{q(n)} S_{i}\right) \leq$ $\nu^{B}\left(\bigcup_{i=1}^{n} T_{i}\right)<\nu^{B}\left(\bigcup_{i=1}^{q(n)+1} S_{i}\right)$. The mapping $\nu^{B}$ being additive, we may write $q(n) \nu^{B}(S) \leq n \nu^{B}(T)<(q(n)+1) \nu^{B}(S)$ and

$$
\frac{q(n)}{n} \nu^{B}(S) \leq \nu^{B}(T)<\frac{q(n)+1}{n} \nu^{B}(S), \forall n \in \mathbb{N}_{0}
$$

so that $\nu^{B}(T)=\lim _{n \rightarrow \infty} \frac{q(n)}{n} \nu^{B}(S)$. Following the same reasoning with any $C \in P_{*}(X)$ with $C \prec A$ instead of $B$ yields $\nu^{C}(T)=\lim _{n \rightarrow \infty} \frac{q(n)}{n} \nu^{C}(S)$. So, $\nu^{B}(T) / \nu^{B}(S)=\nu^{C}(T) / \nu^{C}(S)$. Since this holds for any $S, T \sim A$, this proves that $\nu^{B}(S)=k \nu^{C}(S)$ for some positive constant $k$ and for all $S$ such that $\nu^{A}(S)=0$.

Define $\nu_{A B C}(S)=\left(\nu^{A}(S), \nu^{B}(S), \nu^{C}(S)\right)$ for all $\left.S \in P(X)\right\}$. Then $\left\{x \in R^{3}\right.$ : $\left.x_{1}=0\right\} \cap \nu_{A B C}(P(X))$ is contained in the ray $\{(0, k t, t): t \geq 0\}$. Since $A \in P_{*}(X)$, 
there is $S$ such that $S \succ A$ or $S \prec A$, whence the set $\left\{x \in \nu_{A B C}(P(X)): x_{1} \neq 0\right\}$ is not empty. We can therefore select vectors $x^{0}, x^{1} \in \nu_{A B C}(P(X))$ such that $x_{1}^{0}=0$ and $x_{1}^{1} \neq 0$. Let $S^{0}$ and $S^{1}$ be such that $\nu_{A B C}\left(S^{0}\right)=x^{0}$ and $\nu_{A B C}\left(S^{1}\right)=x^{1}$.

We show that these two vectors, together, $\operatorname{span} \nu_{A B C}(P(X))$. Let $x \in \nu_{A B C}(P(X))$, with $\nu_{A B C}(S)=x$. We proceed by cases, assuming $x_{1}^{1}>0$ (the case $x_{1}^{1}<0$ being symmetric).

1. Suppose $x_{1}=0$. Since $\left\{x \in R^{3}: x_{1}=0\right\} \cap \nu_{A B C}(P(X))$ is contained in the ray $\{(0, k t, t): t \geq 0\}$, we have $x=k x^{0}$.

2. Suppose $x_{1}>0$. By Richness, there is $T: T \cup S^{1} \sim S^{0}$. Hence, $\nu^{A}(T)=$ $-\nu^{A}\left(S^{1}\right)$. By Richness, there is $R: R \cup T \sim S^{0}, R \sim S$. Hence, $\nu^{A}(R)=$ $\nu^{A}\left(S^{1}\right)$. Since $R \sim S \succ A, B, C$, we know that $\nu^{C}(R)=\alpha \nu^{A}(R)$ and $\nu^{C}(S)=\alpha \nu^{A}(S)$ for some $\alpha \in R$. For the same reason, $\nu^{B}(R)=\beta \nu^{A}(R)$ and $\nu^{B}(S)=\beta \nu^{A}(S)$ for some $\beta \in R$. So, $\nu^{C}(R) / \nu^{C}(S)=\nu^{A}(R) / \nu^{A}(S)$ and $\nu^{B}(R) / \nu^{B}(S)=\nu^{A}(R) / \nu^{A}(S)$. In other words, $\nu_{A B C}(R)$ and $\nu_{A B C}(S)$ are in the same ray and $\nu_{A B C}(S)=\gamma \nu_{A B C}(R)$ for some $\gamma \in R$.

Since $T \cup S^{1} \sim S^{0}$, we know that $\nu_{A B C}\left(T \cup S^{1}\right)$ is in the same ray as $x^{0}$. So, $\nu_{A B C}\left(T \cup S^{1}\right)=\nu_{A B C}(T)+x^{1}=\lambda x^{0}$ for some $\lambda>0$. Similarly, since $T \cup R \sim S^{0}$, we know that $\nu_{A B C}(T \cup R)$ is in the same ray as $x^{0}$. So, $\nu_{A B C}(T \cup R)=\nu_{A B C}(T)+\nu_{A B C}(R)=\lambda x^{0}-x^{1}+\nu_{A B C}(R)=\lambda^{\prime 0}$ for some $\lambda^{\prime}>0$. Whence $\nu_{A B C}(R)=\lambda^{\prime 0}-\lambda x^{0}+x^{1}$. We can therefore write $\nu_{A B C}(S)=\gamma\left(\lambda^{\prime 0}-\lambda x^{0}+x^{1}\right)$. This proves that $x$ is spanned by $x^{0}$ and $x^{1}$.

3. Suppose $x_{1}<0$. By Richness, there is $T: T \cup S \sim S^{0}$ and, hence, $\nu_{A B C}(T \cup S)$ is in the same ray as $x^{0}$. So, $\nu_{A B C}(T \cup S)=\nu_{A B C}(T)+x=\lambda x^{0}$ for some $\lambda>0$. So, $x=\lambda x^{0}-\nu_{A B C}(T)$. In other words, $x$ is spanned by $x^{0}$ and $\nu_{A B C}(T)$. We have seen in case 2 that $\nu_{A B C}(T)$ is spanned by $x^{0}$ and $x^{1}$. So, actually, $x$ is spanned by $x^{0}$ and $x^{1}$.

So, there are two real numbers $\lambda, \gamma$ such that, for any $S \in \mathcal{P}(X)$,

$$
\nu_{A B C}(S)=\lambda \nu_{A B C}\left(S^{0}\right)+\gamma \nu_{A B C}\left(S^{1}\right) .
$$

In particular, $\nu^{A}(S)=\lambda \nu^{A}\left(S^{0}\right)+\gamma \nu^{A}\left(S^{1}\right)=\gamma \nu^{A}\left(S^{1}\right)$ because $\nu^{A}\left(S^{0}\right)=0$. So, $\gamma=\nu^{A}(S) / \nu^{A}\left(S^{1}\right)$. From (15), we also derive $\nu^{C}(S)=\lambda \nu^{C}\left(S^{0}\right)+\gamma \nu^{C}\left(S^{1}\right)$ which yields $\lambda=\left(\nu^{C}(S)-\gamma \nu^{C}\left(S^{1}\right)\right) / \nu^{C}\left(S^{0}\right)$. From (15), we finally derive $\nu^{B}(S)=$ $\lambda \nu^{B}\left(S^{0}\right)+\gamma \nu^{B}\left(S^{1}\right)$. Substituting $\lambda$ and $\gamma$ in this equation yields:

$$
\nu^{B}(S)=\frac{\nu^{C}(S)-\left(\nu^{A}(S) / \nu^{A}\left(S^{1}\right)\right) \nu^{C}\left(S^{1}\right)}{\nu^{C}\left(S^{0}\right)} \nu^{B}\left(S^{0}\right)+\nu^{A}(S) \nu^{B}\left(S^{1}\right) / \nu^{A}\left(S^{1}\right),
$$

showing that $\nu^{B}$ is a linear combination of $\nu^{A}$ and $\nu^{C}$.

Hence, for every $A, B, C \in P_{*}(X)$, such that none of them are indifferent, there are two real numbers $\alpha, \beta$ such that $\nu^{A}=\alpha \nu^{B}+\beta \nu^{C}$. Consider now $A, B$ and $C$ such that $B \nsim C$. Using richness, we select $D$ and $D^{\prime}$ not indifferent to any of 
$A, B$ or $C$ and such that $D \nsim D^{\prime}$. We can express each of $\nu^{A}, \nu^{B}, \nu^{C}$ as a linear combination of $\nu^{D}$ and $\nu^{D^{\prime}}$. For instance,

$$
\begin{aligned}
& \nu^{A}=\alpha_{A} \nu^{D}+\beta_{A} \nu^{D^{\prime}}, \\
& \nu^{B}=\alpha_{B} \nu^{D}+\beta_{B} \nu^{D^{\prime}} \\
& \nu^{C}=\alpha_{C} \nu^{D}+\beta_{C} \nu^{D^{\prime}} .
\end{aligned}
$$

From (17) and (18), we derive

$$
\nu^{D}=\frac{\nu^{C} \beta_{B}-\nu^{B} \beta_{C}}{\alpha_{C} \beta_{B}-\alpha_{B} \beta_{C}}
$$

and

$$
\nu_{D^{\prime}}=\frac{\nu^{C} \alpha_{B}-\nu^{B} \alpha_{C}}{\beta_{C} \alpha_{B}-\beta_{B} \alpha_{C}} .
$$

We substitute $\nu^{D}$ and $\nu^{D^{\prime}}$ in (16) and we obtain that $\nu^{A}$ is a linear combination of $\nu^{B}$ and $\nu^{C}$. This suffices to show the entire space $\left\{\nu^{E}: E \in P_{*}(X)\right\}$ can be spanned by any two of its members $\nu^{B}, \nu^{C}$ with $B \nsim C$, since the selection of $A, B, C$ in the proof was arbitrary.

\subsection{Lemma 10}

We consider two cases.

(1) $\nu^{B}(D)=k \nu^{C}(D)$ for for some $k \in \mathbb{R}_{++}$and all $D \in \mathcal{P}(X)$. Then $\nu^{A}(D)=$ $-\lambda \nu^{B}(D)$ for any $D$. But this is not possible because, by Lemma 7 , we know that, for any $D \prec B$, we have $\nu^{B}(D)<0$ and $\nu^{A}(D)<0$. The cases $\nu^{A}=k \nu^{C}$ and $\nu^{A}=k \nu^{B}$ are treated in the same way.

(2) $A \succ B \succ C$ (the 5 other orderings are treated in the same way).

By Lemma $9, \nu^{A}$ and $\nu^{B}$ span $\left\{\nu^{E}: E \in \mathcal{P}_{*}(X)\right\}$. For every $C \in \mathcal{P}_{*}(X)$, let $\alpha(C)$ and $\beta(C)$ be the solution of $\nu^{C}=\alpha(C) \nu^{A}+\beta(C) \nu^{B}$. Since $C \prec B$, we must have $\alpha(C)<0$ or $\beta(C)<0$ as assuming otherwise would imply, for any set $S$ such that $C \prec S \prec B$, that it is impossible to have $\nu^{C}(S)>0$. We must also have $\beta(C)>0$ because $\nu^{C}(A)$ must be positive. Hence, we must have $\alpha(C)<0<\beta(C)$. Assume by contradiction that $-\lambda \nu^{A}=\delta \nu^{B}+(1-\delta) \nu^{C}$ for some $\lambda \in \mathbb{R}_{++}$and $\delta \in] 0,1[$. This implies that

$$
\nu^{C}=\frac{\lambda}{\delta-1} \nu^{A}+\frac{\delta}{\delta-1} \nu^{B}
$$

with $\delta /(\delta-1)<0$, a contradiction of the fact that $0<\beta(C)$.

\subsection{Lemma 11}

Consider two sets $B$ and $C \in \mathcal{P}_{*}(X)$ such that $C \prec B$. Since $\nu^{B}$ and $\nu^{C}$ are linearly independent, they span by Lemma 9 , the set $\left\{\nu^{E}: E \in \mathcal{P}_{*}(X)\right\}$. For every $A \in \mathcal{P}_{*}(X)$, let $\alpha(A)$ and $\beta(A)$ be the solution of $\nu^{A}=\alpha(A) \nu^{B}+\beta(A) \nu^{C}$. If 
$A \prec B, C$, then we must have $\alpha(A)<0$ or $\beta(A)<0$. Indeed, assuming otherwise would imply, for any set $S$ such that $A \prec S \prec B, C$, that it is impossible to have $\nu^{A}(S)>0$. Simultaneously, we must also have $\beta(A)>0$ because $\nu^{A}(B)$ must be positive. So, we must have $\alpha(A)<0<\beta(A)$. Define the function $\rho: \mathcal{P}(X) \longrightarrow \mathbb{R}$ by $\rho(A)=-\beta(A) / \alpha(A)$ for any set $A$. We have $\rho(A)>0$ for all $A \in \mathcal{P}_{*}(X)$ such that $A \prec C$.

We now show that, for all $A^{\prime} \in \mathcal{P}_{*}(X)$ such that $A^{\prime} \prec A \prec C$, one has $\rho\left(A^{\prime}\right)<\rho(A)$ so that the function $\rho$ numerically represent the ranking of decisions that are worse than $A$. Suppose to the contrary that $\rho\left(A^{\prime}\right) \geq \rho(A)$. Since $\nu^{A}(A)=\alpha(A) \nu^{B}(A)+\beta(A) \nu^{C}(A)=0$, we have:

$$
-\frac{\beta(A)}{\alpha(A)}=\rho(A)=\frac{\nu^{B}(A)}{\nu^{C}(A)} \leq-\frac{\beta\left(A^{\prime}\right)}{\alpha\left(A^{\prime}\right)}=\rho\left(A^{\prime}\right) .
$$

Hence $\nu^{B}(A) \alpha\left(A^{\prime C}(A) \beta\left(A^{\prime}\right)\right.$ and $\nu^{A^{\prime}}(A)=\nu^{B}(A) \alpha\left(A^{\prime C}(A) \beta\left(A^{\prime}\right) \leq 0\right.$, which implies $A \prec A^{\prime}$. A contradiction. Notice that the converse is also true. Hence, for all $A, A^{\prime} \in \mathcal{P}_{*}(X)$ satisfying $A, A^{\prime} \prec C, A^{\prime} \precsim A \Longleftrightarrow \rho\left(A^{\prime}\right) \leq \rho(A)$.

Similarly, it is easy to prove that, for all sets $A$ and $A^{\prime} \in \mathcal{P}_{*}(X)$ such that $A$, $A^{\prime} \succ B$, it is the case that $\rho(A)>0$ and $A^{\prime} \precsim A \Longleftrightarrow \rho\left(A^{\prime}\right) \leq \rho(A)$.

Define now the set $Q=\left\{\rho(A): A \in \mathcal{P}_{*}(X), A \prec C\right\}$. This set has a greatest lower bound $\rho^{*} \geq 0$ (because $\rho(A)>0$ for all set $A \in \mathcal{P}(X)$ such that $A \prec C$ ). We can actually show that $\rho^{*}>0$. Indeed, assume by contradiction that $\rho^{*}=0$. Since $B \in \mathcal{P}_{*}(X)$, there exists a set $D \in \mathcal{P}_{*}(X)$ such that $D \succ B$. Because $\rho^{*}=0$, there is also a set $F \in \mathcal{P}_{*}(X)$ with $\rho(F)$ sufficiently close to zero and such that $\nu^{D}, \nu^{B}$ and $\nu^{F}$ are as the functions $\nu^{A}, \nu^{B}$ and $\nu^{C}$ of Lemma 10, which is not possible. Hence we must conclude that $\rho^{*}>0$.

Furthermore $\rho^{*} \notin Q$ because the set $\left\{A \in \mathcal{P}_{*}(X): A \prec C\right\}$ has no minimal element. Let $\mu$ be any of the element in the ray $\left\{x\left(-\nu^{B}+\rho^{*} \nu^{C}\right): x>0\right\}$. To be specific, define $\mu$ by: $\mu=-\nu^{B}+\rho^{*} \nu^{C}$. By construction, $\mu$ belongs to the elements spanned by $\left(\nu^{B}, \nu^{C}\right)$, as per Lemma 9 .

We now prove that $\mu(A)>0$ for all $A \in \mathcal{P}_{*}(X)$. Suppose to the contrary that $\mu(A) \leq 0$ for some $A \in \mathcal{P}_{*}(X)$. By definition of $\mathcal{P}_{*}(X)$, there are decisions $B$ and $C$ such that $A \prec C \prec B$. We know that $\mu(A)=-\nu^{B}(A)+\rho^{*} \nu^{C}(A) \leq 0$. Hence, it follows that $\rho^{*} \nu^{C}(A) \leq \nu^{B}(A)$ and $\rho^{*} \geq \nu^{B}(A) / \nu^{C}(A)$ because $\nu^{C}(A)<0$. Since $\nu^{A}(A)=\alpha(A) \nu^{B}(A)+\beta(A) \nu^{C}(A)=0$, we have $\rho^{*} \geq-\beta(A) / \alpha(A)$, which is impossible because $\rho^{*} \notin Q$. Hence $\mu(A)>0$ for all $A \in \mathcal{P}_{*}(X)$.

Finally, we notice that the function $\mu$ is additive for disjoint sets because it is the linear combination of two functions that are themselves additives on disjoint sets.

\subsection{Lemma 12}

Pick any set $C \in \mathcal{P}_{*}(X)$ and, for every set $A \in \mathcal{P}_{*}(X)$, let $\alpha(A)$ and $\beta(A)$ be the solution of $\nu^{A}=\alpha(A) \nu^{C}+\beta(A) \mu$. These $\alpha(A)$ and $\beta(A)$ exist because $\nu^{C}$ and $\mu$ are linearly independent and, by 9 , can span the whole set $\left\{\nu^{E}: E \in \mathcal{P}_{*}(X)\right\}$. 
By construction, $\nu^{A}(A)=0=\alpha(A) \nu^{C}(A)+\beta(A) \mu(A)$ or, equivalently, $\frac{\nu^{C}(A)}{\mu(A)}=$ $\frac{-\beta(A)}{\alpha(A)}$. Hence, in order to show that $\nu^{C} / \mu$ is a numerical representation of $\succsim$ on $\mathcal{P}_{*}(X)$, it suffices to show that $-\beta / \alpha$ represents $\succsim$ on $\mathcal{P}_{*}(X)$. Notice first that $-\beta / \alpha$ is well-defined because $-\beta(A) / \alpha(A)=\nu^{C}(A) / \mu(A)$ and $\mu(A)>0$ for all $A \in$ $\mathcal{P}_{*}(X)$. Pick any two decisions $A$ and $B \in \mathcal{P}_{*}(X)$ such that $A \succsim B$. By construction, $\nu^{B}(A) \geq 0$. Hence, we must have $\alpha(B) \nu^{C}(A)+\beta(B) \mu(A) \geq 0$ and $\nu^{C}(A) \geq$ $-\beta(B) \mu(A) / \alpha(B)$. We also have $\alpha(A) \nu^{C}(A)+\beta(A) \mu(A)=0$ or, equivalently, $\nu^{C}(A)=-\beta(A) \mu(A) / \alpha(A)$. Hence, $-\beta(A) \mu(A) / \alpha(A) \geq-\beta(B) \mu(A) / \alpha(B)$ or, after simplification, $-\beta(A) / \alpha(A) \geq-\beta(B) / \alpha(B)$. We have therefore proved that $A \succsim B$ implies $-\beta(A) / \alpha(A) \geq-\beta(B) / \alpha(B)$. Proving the converse is easily done by just reverting the argument.

\subsection{Lemma 13}

Consider any set $C \in \mathcal{P}_{*}(X)$. For any sets $A$ and $B \in \mathcal{P}_{*}(X), A \sim_{l} B$ implies, by Lemma 5, that $A \cup D \sim B \cup D$ for some $D \prec A$ such that $D \cap(A \cup B)=\varnothing$. Since, thanks to Lemma $16, \nu^{C} / \mu$ numerically represents the ordering $\succsim$ on $\mathcal{P}_{*}(X)$, one has:

$$
\frac{\nu^{C}(A \cup D)}{\mu(A \cup D)}=\frac{\nu^{C}(B \cup D)}{\mu(B \cup D)} .
$$

or, using the disjoint-additivity of $\nu^{C}$ and $\mu$ :

$$
\frac{\nu^{C}(A)+\nu^{C}(D)}{\mu(A)+\mu(D)}=\frac{\nu^{C}(B)+\nu^{C}(D)}{\mu(B)+\mu(D)} .
$$

Moreover, since the statement $A \sim_{l} B$ is constructed from the statement that $A \sim$ $B \sim E$ for some set $E$, it follows from the fact that $\nu^{C} / \mu$ numerically represents the ordering $\succsim$ that:

$$
\frac{\nu^{C}(A)}{\mu(A)}=\frac{\nu^{C}(B)}{\mu(B)}
$$

or, equivalently:

$$
\nu^{C}(A)=\frac{\mu(A) \nu^{C}(B)}{\mu(B)} .
$$

Substituting equality (20) into equality (19) yields:

$$
\frac{\nu^{C}(B) \mu(A) / \mu(B)+\nu^{C}(D)}{\mu(A)+\mu(D)}=\frac{\nu^{C}(B)+\nu^{C}(D)}{\mu(B)+\mu(D)}
$$

or, after some simplifications and rearranging:

$$
\left(\nu^{C}(B) \mu(D)-\mu(B) \nu^{C}(D)\right][\mu(A)-\mu(B)]=0 .
$$

If $\mu(A)-\mu(B) \neq 0$, then $\nu^{C}(B) / \mu(B)=\nu^{C}(D) / \mu(D)$ and $B \sim D$, which is incompatible with the definition of $D$. We therefore conclude that $\mu(A)-\mu(B)=0$ and, hence, $\mu(A)=\mu(B)$ and $\nu^{C}(A)=\nu^{C}(B)$. 


\subsection{Lemma 14}

Take any reference set $C \in \mathcal{P}_{*}(X)$ and consider any set $D \in \mathcal{P}_{*}(X)$. By richness, there are sets $A$ and $B \in \mathcal{P}_{*}(X)$ such that $D \succ A \succ B$. By Lemma 2, there is a set $A^{\prime}$ such that $A^{\prime} \sim A$ and $A^{\prime} \cap B=\varnothing$. By Averaging, $A \succ A^{\prime} \cup B \succ B$. By Richness, there are sets $A_{1}, A_{2}, \ldots$ such that, for $i \in\{1,2, \ldots\}, A \sim A_{i}, A_{i} \cap\left(A \bigcup_{j=1}^{i-1} A_{j}\right)=$ $\varnothing$ and $A_{i} \cup B \sim A^{\prime} \cup B$. By Lemma 13, $\nu^{C}\left(A_{i}\right)=\nu^{C}(A)$ and $\mu\left(A_{i}\right)=\mu(A)$ for $i \in\{1,2, \ldots\}$. Some of the sets $A_{1}, A_{2}, \ldots$ may intersect with $D$, but the number of such intersecting sets is necessarily finite (as these sets are pairwise disjoint). So, if we drop them, we can still end up with an infinite collection of sets $A_{1}, A_{2}, \ldots$ that are all disjoint from $D$. We therefore assume hereafter that $A_{i} \cap D=\varnothing$ for $i \in\{1,2, \ldots\}$. By Averaging, $D \succ D \bigcup_{j=1}^{k} A_{j} \succ A$, for any $k \in\{1,2, \ldots\}$. By Richness, for any $k \in\{1,2, \ldots\}$, there is a set $E$ such that $E \cap(A \cup D)=\varnothing, E \sim D$ and $E \cup A \sim C \bigcup_{j=1}^{k} A_{j}$. By Lemma 12, $\nu^{C}(D) / \mu(D)=\nu^{C}(E) / \mu(E)$ and, for all $i \in\{1,2, \ldots\}$, one has $\nu^{C}(A) / \mu(A)=\nu^{C}\left(A_{i}\right) / \mu\left(A_{i}\right)$ and

$$
\frac{\nu^{C}(E \cup A)}{\mu(E \cup A)}=\frac{\nu^{C}\left(D \bigcup_{j=1}^{i} A_{j}\right)}{\mu\left(D \bigcup_{j=1}^{i} A_{j}\right)} .
$$

Using the disjoint-additivity of $\nu^{C}$ and $\mu$, one can write, for any $k$ :

$$
\frac{\nu^{C}(E)+v^{C}(A)}{\mu(E)+\mu(A)}=\frac{\nu^{C}(D)+\sum_{j=1}^{k} \nu^{C}\left(A_{j}\right)}{\mu(D)+\sum_{j=1}^{k} \mu\left(A_{j}\right)}=\frac{\nu^{C}(D)+k \nu^{C}(A)}{\mu(D)+k \mu(A)}
$$

which can be equivalently written as:

$$
\left(\nu^{C}(E)+\nu^{C}(A)\right)(\mu(D)+k \mu(A))=\left(\nu^{C}(D)+k \nu(A)\right)(\mu(E)+\mu(A)) .
$$

If one substitutes $\nu^{C}(D) \mu(E) / \mu(D)$ for $v^{C}(E)$ in this expression and performs similar manipulation as in the proof of Lemma 13) one obtains:

$$
\left[\nu^{C}(A) \nu^{C}(D)-\mu(A) \mu(D)\right][(\mu(D)-k \mu(E))]=0
$$

One can not have $\left[\nu^{C}(A) \nu^{C}(D)-\mu(A) \mu(D)\right]=0$ because assuming this would amount to assume that $\nu^{C}(A) / \mu(A)=\nu^{C}(D) / \mu(D)$ and, since the function $\nu^{C} / \mu$ numerically represents the ordering $\succsim$, that $A \sim D$, which is not the case. We therefore conclude that $\mu(D)-k \mu(E)=0$ and, hence, $\mu(E)=\mu(D) / k$. For any $\varepsilon>0$, we can therefore guarantee that $\mu(E)<\varepsilon$ by choosing a suitably large $k$.

\subsection{Lemma 15}

If the function $\mu$ of Lemma 11 is such that $\mu(S)>0$ for all set $S \in \mathcal{P}(X)$, we define $\mu_{+}=\mu$ and the proof is done.

Otherwise, we first prove that $\mu(S) \geq 0$ for all $S \in m(X)$. Assume by contradiction that $\mu(S)<0$ for some $S \in m(X)$ and choose (using richness) sets $C$ and $T \in \mathcal{P}_{*}(X)$ satisfying $S \cap T=\varnothing, \nu^{C}(T)<0$ and $\mu(T)$ sufficiently small (thanks 
to Lemma 14). Consider the set $D=T \cup S$ and its numerical representation by the function $v^{C} / \mu$ :

$$
\frac{\nu^{C}(T)+\nu^{C}(S)}{\mu(T)+\mu(S)} .
$$

The numerator of this expression is negative because $S \in m(X)$ and $\nu^{C}(S)<0$ if $\nu^{C}(T)<0$ by definition of the function $v^{C}$ provided in Lemma 7 . For a sufficiently small $\mu(T)$ one can also make the denominator of the expression negative. Hence $\nu^{C}(D) / \mu(D)>0$ and, since the function $v^{C} / \mu$ numerically represents the ordering $\succsim$ on $\mathcal{P}_{*}(X)$, one concludes that $D \succ T$. Yet this contradicts the Averaging axiom according to which $T \succ D=T \cup S$ (because $S \in m(X)$ ).

Using an analogous argument, we can prove that $\mu(S) \geq 0$ for all set $S \in M(X)$.

We now claim that it is impossible to have $\mu(S)=0=\mu(T)$ for some $S \in m(X)$ and some $T \in M(X)$. Assume indeed that $\mu(S)=0=\mu(T)$ for some $S \in m(X)$ and $T \in M(X)$. Remember from Lemma 1 that $S \cap T=\varnothing$. By Averaging $S \cup T \in \mathcal{P}_{*}(X)$ and, as a result, one has $\mu(S \cup T)>0$ by Lemma 9 . Yet, using the disjoint-additivity of $\mu$, we find that $\mu(S \cup T)=0$ although $S \cup T \in \mathcal{P}_{*}(X)$. This contradiction shows the impossibility of having $\mu(S)=0=\mu(T)$ for some $S \in m(X)$ and some $T \in M(X)$.

Suppose now that $\mu(S)=0$ for some $S \in m(X)$. This implies $\mu(T)>0$ for all $T \in \mathcal{P}_{*}(X) \cup M(X)$. We know from Lemma 11 that $\mu=-\nu^{B}+\rho^{*} \nu^{C}$ for some sets $B$ and $C \in \mathcal{P}_{*}(X)$. If we choose a number $\rho_{+}<\rho^{*}$ and we define $\mu_{+}=-\nu^{B}+\rho_{+} \nu^{C}$, we are sure that $\mu(S)>0$. If, in addition, we choose the number $\rho_{+}$to be as close as necessary to $\rho^{*}$, we can guarantee that $\mu(T)>0$ for all $T \in \mathcal{P}_{*}(X)$. The mapping $\mu_{+}$is clearly disjoint-additive and can be spanned by two (linearly independent) element of the family $\left\{\nu^{E}: E \in \mathcal{P}_{*}(X)\right\}$. We still have to prove that $\mu_{+}(T)>0$ for all $T \in m(X)$. If $T \neq S$ and $\mu(T)>0$, then the proof is obvious because we have chosen $\rho_{+}$to be very close to $\rho^{*}$. If $T \neq S$ and $\mu(T)=0$, one must remember that $\mu(T)=-\nu^{B}(T)+\rho^{*} \nu^{C}(T)$, where $\nu^{B}(T)<0$ and $\nu^{C}(T)<0$. Hence if we choose $\rho_{+}<\rho^{*}$, then $\mu_{+}(T)=-\nu^{B}(T)+\rho_{+} \nu^{C}(T)$ is necessarily larger that $\mu(T)$ and, hence, positive.

The case where $\mu(S)=0$ for some $S \in M(X)$ can be handled in a similar fashion

\subsection{Lemma 16}

For every set $E \in \mathcal{P}_{*}(X)$, let $\alpha(E)$ and $\beta(E)$ be the solution of the equation $\nu^{E}=$ $\alpha(E) \nu^{C}+\beta(E) \mu_{+}$. As in the proof of Lemma 12, the existence of these real numbers $\alpha(E)$ and $\beta(E)$ is secured by the fact that $\nu^{C}$ and $\mu_{+}$are linearly independent and, thanks to Lemma 9, can span the whole set $\left\{\nu^{E}: E \in \mathcal{P}_{*}(X)\right\}$. By construction, $\nu^{E}(E)=0=\alpha(E) \nu^{C}(E)+\beta(E) \mu_{+}(E)$ or, equivalently, $\frac{\nu^{C}(E)}{\mu_{+}(E)}=\frac{-\beta(E)}{\alpha(E)}$. As in the proof of Lemma 12 again, the proof that $\nu / \mu_{+}$is a numerical representation of $\succsim$ on $\mathcal{P}_{*}(X)$ amounts to showing that $-\beta / \alpha$ represents $\succsim$ on $\mathcal{P}_{*}(X)$. Notice first that $-\beta / \alpha$ is well-defined because $-\beta(E) / \alpha(E)=\nu^{C}(E) / \mu_{+}(E)$ and $\mu_{+}(E)>0$ for all $E \in \mathcal{P}_{*}(X)$. Consider any two sets $A$ and $B \in \mathcal{P}_{*}(X)$ with $A \succsim B$. From Lemma $7, \nu^{B}(A) \geq 0$. Hence one has $\alpha(B) \nu^{C}(A)+\beta(B) \mu_{+}(A) \geq 0$ and $\nu^{C}(A) \geq$ 
$-\beta(B) \mu_{+}(A) / \alpha(B)$. One also has $\alpha(A) \nu^{C}(A)+\beta(A) \mu_{+}(A)=0$ or, equivalently, $\nu^{C}(A)=-\beta(A) \mu_{+}(A) / \alpha(A)$. Hence $-\beta(A) \mu_{+}(A) / \alpha(A) \geq-\beta(B) \mu_{+}(A) / \alpha(B)$ or, after simplification, $-\beta(A) / \alpha(A) \geq-\beta(B) / \alpha(B)$. We have therefore proved that $A \succsim B$ implies $-\beta(A) / \alpha(A) \geq-\beta(B) / \alpha(B)$. The converse implication is obtained by reversing the argument.

\subsection{Theorem 1}

If the ordering $\succsim$ is trivial, then the numerical representation provided by (1) trivially holds with $u$ constant. We therefore assume in the rest of the proof that $\succsim$ is not trivial.

Take any reference set $C$ in $\mathcal{P}_{+}(X)$. Just as in equation (4) preceding Lemma 12, define the function $f_{+}^{C}: \mathcal{P}(X) \rightarrow \mathbb{R}$ by $f_{+}^{C}(A)=\frac{\nu^{C}(A)}{\mu_{+}(A)}$ for every $A \in \mathcal{P}(X)$. Since $\nu^{C}$ and $\mu_{+}$are both set disjoint-additive, one can write:

$$
f_{+}^{C}(A)=\frac{\sum_{a \in A} \nu^{C}(\{a\})}{\sum_{a \in A} \mu_{+}(\{a\})}=\frac{\sum_{a \in A} f_{+}^{C}(\{a\}) \mu_{+}(\{a\})}{\sum_{a \in A} \mu_{+}(\{a\})} .
$$

Define the two functions $u: X \rightarrow \mathbb{R}$ and $p: X \rightarrow \mathbb{R}^{++}$by $u(a)=f(\{a\})$ and $p(a)=\mu_{+}(\{a\})$. One has:

$$
f_{+}^{C}(A)=\frac{\sum_{a \in A} u(a) p(a)}{\sum_{a \in A} p(a)} .
$$

We already know from Lemma 16) that $A \succsim B \Longleftrightarrow f_{+}^{C}(A) \geq f_{+}^{C}(B)$ for all decisions $A$ and $B \in \mathcal{P}_{*}(X)$. We only need to prove that the equivalence must hold also for decisions $A$ and $B \in \mathcal{P}(X)$ that can be maximal or minimal in that set. We consider several cases.

1. $A \in M(X)$ and $B \in \mathcal{P}_{*}(X)$. By Lemma 2 , there is $B^{\prime} \in \mathcal{P}(X)$ such that $B^{\prime} \cap A=\varnothing$ and $B^{\prime} \sim B$. By Lemma 16, $\nu^{C}(B) / \mu_{+}(B)=\nu^{C}\left(B^{\prime}\right) / \mu_{+}\left(B^{\prime}\right)$. By Averaging, $A \succ A \cup B^{\prime} \succ B^{\prime}$ and, hence, $A \cup B^{\prime} \in \mathcal{P}_{*}(X)$. We therefore have:

$$
\frac{\nu^{C}\left(A \cup B^{\prime}\right)}{\mu_{+}\left(A \cup B^{\prime}\right)}=\frac{\nu^{C}(A)+\nu^{C}\left(B^{\prime}\right)}{\mu_{+}(A)+\mu_{+}\left(B^{\prime}\right)}>\frac{\nu^{C}\left(B^{\prime}\right)}{\mu_{+}\left(B^{\prime}\right)}=\frac{\nu^{C}(B)}{\mu_{+}(B)} .
$$

Since $\mu_{+}$is always strictly positive, this yields

$$
\frac{\nu^{C}(A)}{\mu_{+}(A)}>\frac{\nu^{C}(B)}{\mu_{+}(B)}
$$

a statement that is in line with the fact that $A \succ B$.

2. $A \in m(X)$ and $B \in \mathcal{P}_{*}(X)$. Similar to the previous case.

3. $A, B \in m(X)$. Choose a decision $D \in \mathcal{P}_{*}(X)$ in such a way that $D \cap(A \cup B)=$ $\varnothing$. By Averaging, $B \cup D \succ B$ and, by transitivity, $B \cup D \succ A$. Using the 
result of the proof of case $2, \nu^{C}(B \cup D) / \mu_{+}(B \cup D)>\nu^{C}(A) / \mu_{+}(A)$ and

$$
\frac{\nu(B)+\nu(C)}{\mu(B)+\mu(C)}>\frac{\nu(A)}{\mu(A)} \text {. }
$$

By Lemma 14 , we can choose $D$ in a given equivalence class of $\succsim$, with $\mu_{+}(C)$ as close to zero as required. Since all sets in a given equivalence class have the same ratio $\nu^{C} / \mu$, we can actually choose $D$ in such a way that both $\mu_{+}(D)$ and $\nu^{C}(D)$ are arbitrarily close to zero. Assume now by contradiction that $f_{+}^{C}(A)>f_{+}^{C}(B) \Longleftrightarrow \nu^{C}(A) / \mu_{+}(A)>\nu^{C}(B) / \mu_{+}(B)$. Then ,if we choose the set $D$ as described above, we clearly have

$$
\frac{\nu^{C}(A)}{\mu_{+}(A)}>\frac{\nu^{C}(B)+\nu^{C}(D)}{\mu_{+}(B)+\mu_{+}(D)},
$$

which contradicts $(21)$.

4. $A, B \in M(X)$. Similar to the previous case.

5. $A \in M(X)$ and $B \in m(X)$. We know from Lemma 1 that $A \cap B=\varnothing$. Then $A \succ A \cup B \succ B$ by averaging and, hence, $A \cup B \in \mathcal{P}_{*}(X)$. From $A \succ A \cup B$ and case 1 , we derive $f_{+}^{C}(A)>f_{+}^{C}(A \cup B)$. From $A \cup B \succ B$ and case 2, we derive $f_{+}^{C}(A \cup B)>f_{+}^{C}(B)$ and the required conclusion $f(A)>f(B)$ follows from transitivity.

\subsection{Proposition 1}

Suppose that $A, B$ and $C$ are three finite and non-empty subsets of $\mathbb{R}$ such that $A \succ B \succ C$ or $A \prec B \prec C$. Richness implies the existence of a set $D$ disjoint from $A$ and $C$ such that $A \sim D$ and $D \cup C \sim B$. For any set $E \in \mathcal{P}(X)$, define $U(E)$ by

$$
U(E)=\frac{\sum_{e \in E} p(e) u(e)}{\sum_{e \in E} p(e)}
$$

Then:

$$
U(D)=\frac{\sum_{d \in D} p(d) u(d)}{\sum_{d \in D} p(d)}=U(A)
$$

and:

$$
U(D \cup C)=\frac{\sum_{d \in D} p(d) u(d)+\sum_{c \in C} p(c) u(c)}{\sum_{d \in D} p(d)+\sum_{c \in C} p(c)}=U(B) .
$$

This last equation can be rewritten as

$$
\sum_{d \in D} p(d) u(d)+\sum_{c \in C} p(c) u(c)=U(B)\left(\sum_{d \in D} p(d)+\sum_{c \in C} p(c)\right) .
$$

From (22), we obtain $\sum_{d \in D} p(d) u(d)=U(A) \sum_{d \in D} p(d)$. By definition of $U$, we also have $\sum_{c \in C} p(c) u(c)=U(C) \sum_{c \in C} p(c)$. If we replace in (23), we find:

$$
U(A) \sum_{d \in D} p(d)+U(C) \sum_{c \in C} p(c)=U(B)\left(\sum_{d \in D} p(d)+\sum_{c \in C} p(c)\right) .
$$


or:

$$
(U(A)-U(B))\left(\sum_{d \in D} p(d)\right)=(U(B)-U(C))\left(\sum_{c \in C} p(c)\right)
$$

which amounts to:

$$
\frac{U(A)-U(B)}{U(B)-U(C)}=\frac{\sum_{c \in C} p(c)}{\sum_{d \in D} p(d)} .
$$

Since this holds for any sets $A, B$ and $C$, it holds in particular for $B=\{b\}$. Thanks to the continuity of $u$, we can choose $b$ so that $U(B)=u(b)$ is between $U(C)$ and $U(A)$ and is as close as we want to $U(A)$ or $U(C)$. We can therefore make the ratio in the left-hand side of $(24)$ as close to 0 or $\infty$ as we wish. Hence, for given $A$ and $C$, Richness implies the existence of a set $D$ with $\sum_{d \in D} p(d)$ arbitrary close to 0 or to $\infty$.

Suppose $p$ is non-decreasing. If we want to make $\sum_{d \in D} p(d)$ arbitrary close to 0 , then $\max _{d \in D} p(d)$ must be arbitrary close to 0 . This implies that $\lim _{x \rightarrow \inf X} p(x)=$ 0 and, hence, $\max _{d \in D} d$ must be arbitrary close to inf $X$.

- If $u$ is non-decreasing, then $U(D)<U(A)$ (if we have chosen $A \succ C$ ). This contradicts $(22)$ and proves that $u$ continuous and non-decreasing is not compatible with $p$ non-decreasing.

- If $u$ is non-increasing, then $U(D)>U(A)$ (if we have chosen $A \prec C$ ). This contradicts $(22)$ and proves that $u$ continuous and non-increasing is not compatible with $p$ non-decreasing.

Suppose $p$ is non-increasing. If we want to make $\sum_{d \in D} p(d)$ arbitrary close to $\infty$, then $\min _{d \in D} p(d)$ must be arbitrary large. This implies that $\lim _{x \rightarrow \sup X} p(x)=\infty$ and, therefore, $\min _{d \in D} d$ must be arbitrary close to $\sup X$.

- If $u$ is non-decreasing, then $U(D)>U(A)$ (if we have chosen $A \prec C$ ). This contradicts $(22)$ and proves that $u$ continuous and non-decreasing is not compatible with $p$ non-increasing.

- If $u$ is non-increasing, then $U(D)<U(A)$ (if we have chosen $A \succ C$ ). This contradicts $(22)$ and proves that $u$ continuous and non-increasing is not compatible with $p$ non-increasing.

\subsection{Proposition 2}

Assume that $\succsim$ is a continuous UEU ordering so that the $p$ function of expression (1) is a constant function. Hence, for any two sets $S$ and $S^{\prime} \in \mathcal{P}(X)$, one has:

$$
S \succsim S^{\prime} \Longleftrightarrow \sum_{s \in S} \frac{u(s)}{\# S} \geq \sum_{s^{\prime} \in S^{\prime}} \frac{u(s)}{\# S^{\prime}}
$$

For some continuous function $u$. For any set $S$, let $U E U(S)=\sum_{s \in S} \frac{u(s)}{\# S}$. Since $\succsim$ is not trivial there are consequences $a$ and $c \in X$ such that $u(a)>u(c)$. Let $D$ be a set 
such that $D \sim\{a\}$. The set $D$ can be a singleton $(D=\{d\}$ with $u(d)=u(a))$ or a set with several elements. If $D$ is a singleton, then $U E U(D \cup C)=(u(a)+u(c)) / 2$. If $D$ is not a singleton, then $U E U(D \cup C)>(u(a)+u(c)) / 2$. Hence, for all sets $D \sim A, U E U(D \cup C) \geq(u(a)+u(c)) / 2$. The continuity of $u$ implies that, for any real number $\alpha$ between $u(a)$ and $u(c)$, there exists some $B=\{b\} \in \mathcal{P}(X)$ such that $u(b)=\alpha$. If $\alpha$ is chosen to be strictly smaller than $(u(a)+u(c)) / 2$, then $(D \cup C)>(B)$ and $D \cup C \succ B$, for any $D$ with $D \sim A$. Hence, Richness does not hold.

\section{References}

Ahn, D. S. (2008): "Ambiguity without a State Space," Review of Economic Studies, 75, 3-28.

Anscombe, F., and R. Aumann (1963): "A Definition of Subjective Probability," Annals of Mathematical Statistics, 34, 199-205.

Baigent, N., and Y. Xu (2004): "Decisions under complete ignorance," Mimeo,Institute of public economics, Graz university.

Barberà, S., W. Bossert, and P. K. Pattanaik (2004): "Ranking Sets of Objects," in Handbook of Utility Theory, vol. 2: Extensions, ed. by S. Barberà, P. Hammond, and C. Seidl, pp. 893-977. Kluwer, Dordrecht.

Bolker, E. D. (1966): "Functions Resembling Quotient of Measures," Transaction of the American Mathematical Society, 124, 292-312.

(1967): "A Simultaneous Axiomatization of Utility and Subjective Probabilities," Philosophy of Sciences, 34, 333-340.

Broome, J. (1990): "Bolker-Jeffrey Expected Utility Theory and Axiomatic Utilitarianism," Review of Economic Studies, 57, 477-502.

Ellsberg, D. (1961): "Risk, Ambiguity, and the Savage Axioms," Quarterly Journal of Economics, 75, 643-669.

Epstein, L., and J. Zhang (2001): "Subjective Probabilities and Subjectively Unambiguous Events," Econometrica, 69, 265-306.

Ghirartado, P., F. Maccheroni, and M. Marinacci (2004): "Differentiating Ambiguity and Ambiguity Attitude," Journal of Economic Theory, 118, $133-173$.

Ghirartado, P., and M. Marinaccin (2002): "Ambiguity Made Precise: A Comparative Foundation," Journal of Economic Theory, 102, 251-289.

Gravel, N., T. Marchant, and A. Sen (2012): "Uniform Utility Criteria for Decision Making under Ignorance or Objective Ambiguity," Journal of Mathematical Psychology, 56, 297-315. 
Gul, F., And W. Pesendorfer (2001): "Temptation and Self-Control," Econometrica, 69, 1403-1435.

Jeffrey, R. (1965): The Logic of Decision. University of Chicago Press, Chicago, reprint 1983.

Klibanoff, P., M. Marinacci, and S. Mukerji (2005): "A Smooth Model of Decision Making under Ambiguity," Econometrica, 73, 1849-1892.

Krantz, D., R. D. Luce, P. Suppes, and A. Tversky (1971): Foundations of Measurement. Academic Press, New York London.

Meinshausen, M., N. Meinshausen, W. Hare, S. C. B. Raper, K. Frieler, R. Knutti, D. J. Frame, and M. R. Allen (2009): "Greenhouse-gas emission targets for limiting global warming to 2ř c," Nature, $458,1158-1163$.

Nitzan, S., and P. K. Pattanaik (1984): "Median-Based extensions of an Ordering over a Set to the Power Set: An Axiomatic Characterization," Journal of Economic Theory, 34, 252-261.

Olszewski, W. (2007): "Preferences over Sets of Lotteries," Review of Economic Studies, 74, 567-595.

Segal, U. (1987): "The Ellsberg Paradox and Risk Aversion: an Anticipated Utility Approach," International Economic Review, 28, 175-202.

(1990): "Two-stage Lotteris without the Reduction Axiom," Econometrica, 58, 349-377.

WAKker, P. (1988): "The algebraic versus the topological approach to additive representations," Journal of Mathematical Psychology, 32, 421-435. 